\title{
An optimization approach for flow simulations in poro-fractured media with complex geometries
}

\author{
Stefano Berrone ${ }^{1}$ (D) . Alessandro D'Auria ${ }^{1} \cdot$ Stefano Scialò $^{1}$ (i) \\ Received: 9 April 2020 / Accepted: 20 December 2020 / Published online: 2 February 2021 \\ (C) The Author(s) 2021
}

\begin{abstract}
A new discretization approach is presented for the simulation of flow in complex poro-fractured media described by means of the Discrete Fracture and Matrix Model. The method is based on the numerical optimization of a properly defined costfunctional and allows to solve the problem without any constraint on mesh generation, thus overcoming one of the main complexities related to efficient and effective simulations in realistic DFMs.
\end{abstract}

Keywords 3D flows - Darcy flows - Matrix-fracture coupled flows - Optimization methods for elliptic problems . Non-conforming FEM meshes · 2D-3D flow coupling

Mathematics Subject Classification (2010) $65 \mathrm{~N} 30 \cdot 65 \mathrm{~N} 50 \cdot 68 \mathrm{U} 20 \cdot 86-08$

\section{Introduction}

The present work deals with the simulation of the flow in the subsoil, modelled by means of the Discrete Fracture and Matrix (DFM) model. According to this model, underground fractures are represented as planar polygons arbitrarily oriented in a three dimensional porous matrix. The flows considered here are governed by the Darcy law in the three dimensional matrix and by an averaged Darcy law on each fracture plane, with suitable matching conditions at fracture-matrix interfaces and at fracture intersections. The quantity of interest is the hydraulic head, given by the sum of the pressure head and elevation. Single phase stationary flow is considered, with the assumption of continuity of the hydraulic head at both fracture-matrix

Members of the INdAM research group GNCS

Stefano Scialò

stefano.scialo@polito.it

Stefano Berrone

stefano.berrone@polito.it

Alessandro D'Auria

alessandro.dauria@polito.it

1 Dipartimento di Scienze Matematiche, Politecnico di Torino, Corso Duca degli Abruzzi 24, 10129, Torino, Italy interfaces and at fracture-fracture intersections and no longitudinal flow is allowed along fracture intersections. This is a simplified model with respect to other DFM approaches, described, for example in [1] or, more recently, in [2], but still representative of realistic configurations, characterized, e.g., by highly permeable fractures. The main focus of the present work is on geometrical complexity aspects, proposing a problem formulation and a numerical approach suitable for complex and randomly generated networks. The described approach can however be extended to different flow models and different coupling conditions. The geometrical complexity of DFM models mainly arises from the multi-scale nature of the resulting domains and from the presence of multiple intersecting interfaces, where the solution displays an irregular behavior. DFM models are proposed as an alternative to homogenization techniques [3], dual and multy-porosity models [4], or embedded discrete fracture matrix (EDFM) models [5-7], and are characterized by the explicit representation of the underground fractures, dimensionally reduced to planar interfaces into the porous matrix. As a consequence of the random orientation, fractures usually form an intricate system of intersections, with the presence of fractures with very different sizes spanning several orders of magnitude that generate intersections with huge geometrical complexities as, for example, 2D and 3D geometrical objects with very different dimension and objects with enormous aspect ratios. The research on effective numerical tools for DFM simulations 
is particularly active, see e.g. [8-15]. One of the key aspects is the meshing of the domain, with a mesh conforming to the interfaces, suitable for standard approaches for the imposition of interface conditions. The generation of a conforming mesh for realistic fracture networks might, in fact, result in an impossible task, for the extremely high number of geometrical constraints. The mesh conformity constraint at the interfaces can be relaxed by using extended finite elements as suggested, e.g., by [16, 17]. Different approaches are based on the Mimetic Finite Difference method (MFD) [18], as described, for example, in [11, 19], or on Hybrid High Order (HHO) methods as proposed by [20], where a partial non-conformity is allowed between the mesh of the porous medium and of the fractures, or also on Discontinuous Galerkin discretizations, as in [14]. Two or multi-point flux approximation based techniques are described in [21, 22] and gradient schemes in [10]. Virtual Element (VEM) based discretizations have also been recently investigated to ease the mesh generation process in complex DFMs, as in [23] where the VEM is coupled to the Boundary Element method, and in [24], in [25] for poro-elasticity problems, or in [26] where an arbitrary order mixed VEM formulation is proposed.

This work presents a development of an optimizationbased approach, first proposed for Discrete Fracture Networks [27-31] and recently extended to DFM problems in [12]. This approach avoids any mesh conformity requirement for the imposition of interface conditions, which are instead enforced through the minimization of a properly defined cost functional. The computation of the quantities involved in functional definition does not require any constraint on the mesh. Further, the resolution of the optimization problem via a gradient-based scheme allows to de-couple the problems on each fracture and the problem on the porous matrix, thus paving the way for an efficient parallel implementation of the numerical scheme, similarly to what done in $[30,32]$. The discretization scheme described in [12] relies on the Boundary Element Method for the discretization of the problem on three dimensional matrix blocks, thus requiring the splitting of the original three dimensional domain into sub-domains not crossing the fractures, and thus implying a partial mesh conformity at the fracture-matrix interfaces. Here, the three dimensional domain is not split into sub-domains and Finite Elements are used for the discretization of the matrix, on tetrahedral elements that can arbitrarily cross the fractures. Finite elements on triangular meshes are used for the fractures, with elements not conforming to the tetrahedral mesh and also arbitrarily placed with respect to fracture-fracture intersections. The proposed discretization approach thus greatly improves the usability of the method to general DFM geometries, allowing a trivial meshing process of extremely complex domains, thanks to the complete independence of the mesh from all the interfaces.

The structure of the manuscript is the following: Section 2 describes both the classical and the optimization based formulation of the flow problem in a DFM; the following Section 3 describes the derivation of the discrete problem and the proof of its well posedness; Section 4 shows how an equivalent unconstrained optimization problem is derived, and the gradient based scheme used for problem resolution; Section 5 reports some numerical results and finally some conclusions are proposed in Section 6.

\section{Problem description}

This section is devoted to a brief description of the problem of interest, referring to [12] for a more detailed exposition and well posedness results. Let us consider a polyhedral block of porous material, denoted as $\mathcal{D}$, crossed by a fracture network $\Omega$ given by the union of planar polygonal fractures $F_{i}, i=1, \ldots, N_{F}$ in the three-dimensional space, i.e. $\Omega=\bigcup_{i=1}^{N_{F}} F_{i}$. We further denote by $\mathcal{F}$ the set of all fracture indexes. Fractures might intersect, and fracture intersections, also called traces, are indicated as $S_{m}, m=$ $1, \ldots, N_{S}$. We assume, for simplicity, that each trace is given by the intersection of exactly two fractures, such that an injective map $\sigma:\left[1, \ldots, N_{S}\right] \mapsto\left[1, \ldots, N_{F}\right] \times$ $\left[1, \ldots, N_{F}\right]$ can be defined between a trace index and a couple of fracture indexes, as $\sigma(m)=\{i, j\}$ being $S_{m}=$ $\bar{F}_{i} \cap \bar{F}_{j}$. Further, $\mathcal{S}_{i}$ is the set of indexes of all the traces on fracture $F_{i}$ and $\mathcal{S}$ the set of indexes of all the traces in the network. Let us introduce the domain $\tilde{\mathcal{D}}=\mathcal{D} \backslash \bar{\Omega}$, thus given by the original block $\mathcal{D}$ without the internal fractures. Calling $\partial \tilde{\mathcal{D}}$ the boundary of $\tilde{\mathcal{D}}$, let us denote by $\Gamma_{i}^{ \pm}$the portion of $\partial \tilde{\mathcal{D}}$ that matches fracture $F_{i}$, for $i=1, \ldots, N_{F}$, the superscript "+" or "-" referring to one of the two sides of the boundary "around" the fracture (see Fig. 1); the unit normal vector to $\Gamma_{i}^{ \pm}$is $\mathbf{n}_{\Gamma_{i}}^{ \pm}$, always pointing outward from $\tilde{\mathcal{D}}$. A jump operator is introduced for any sufficiently regular vector function $\mathbf{v}$ on $\tilde{\mathcal{D}}$, defined as the jump of $\mathbf{v}$ along the normal direction to the faces $\Gamma_{i}^{ \pm}$:

$[\mathbf{v} \cdot \mathbf{n}]_{\Gamma_{i}}:=\left(\mathbf{v}_{\mid \Gamma_{i}^{+}} \cdot \mathbf{n}_{\Gamma_{i}^{+}}\right)-\left(\mathbf{v}_{\mid \Gamma_{i}^{-}} \cdot \mathbf{n}_{\Gamma_{i}^{+}}\right)$.

Similarly, for $i=1, \ldots, N_{F}$ we denote by $\tilde{F}_{i}$ the fracture $F_{i}$ without traces, i.e. $\tilde{F}_{i}=F_{i} \backslash \bigcup_{m \in \mathcal{S}_{i}} S_{m}$, and for each trace $S_{m}, m \in \mathcal{S}_{i}$, for any sufficiently regular vector function $\mathbf{w}_{i}$ on $F_{i}$, the jump of the normal component of $\mathbf{w}_{i}$ across trace $S_{m}$ on $F_{i}$ is denoted as:

$$
\left[\mathbf{w}_{i} \cdot \mathbf{n}\right]_{S_{m}}:=\left(\mathbf{w}_{i \mid S_{m}^{+}} \cdot \mathbf{n}_{S_{m}}^{i}\right)-\left(\mathbf{w}_{i \mid S_{m}^{-}} \cdot \mathbf{n}_{S_{m}}^{i}\right),
$$




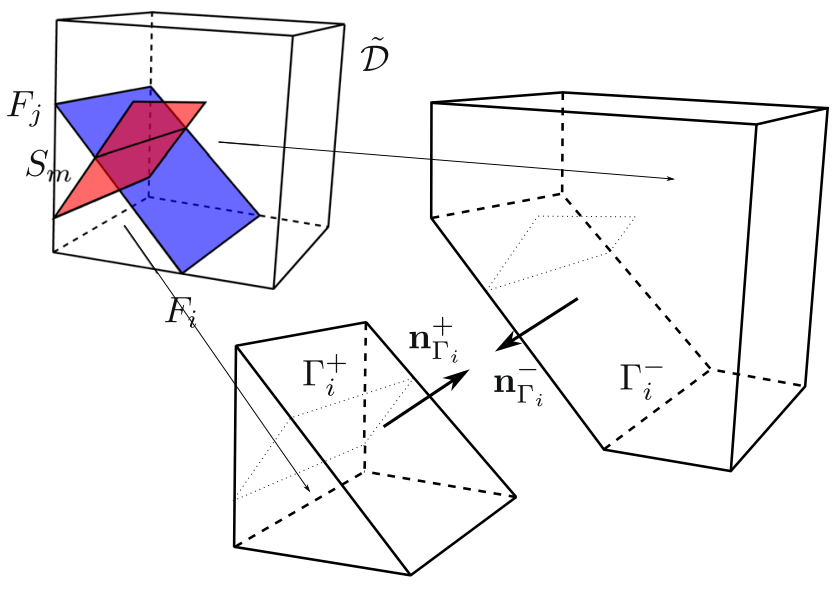

Fig. 1 Nomenclature exemplification

with $S_{m}^{ \pm}$the two sides of the portion of the boundary of $\tilde{F}_{i}$ lying on $S_{m}$ and $\mathbf{n}_{S_{m}}^{i}$ the normal unit vector to $S_{m}$ with a fixed orientation on $F_{i}$. These jump operators are easily extended to functions defined on the whole 3D domain $\mathcal{D}$ and on the whole fractures $F_{i}, i=1, \ldots, N_{F}$, with the \pm superscripts still denoting the two sides of the interface $\Gamma_{i} \equiv F_{i}, \forall i=1, \ldots, N_{F}$, or $S_{m}, \forall m=1, \ldots, N_{S}$.

The portion of $\partial \tilde{\mathcal{D}}$ not matching any fracture is split in a Dirichlet part $\Gamma_{D}$ and a Neumann part $\Gamma_{N}, \Gamma_{D} \cap$ $\Gamma_{N}=\emptyset$, where, for simplicity of exposition, we assume homogeneous Dirichlet and Neumann boundary conditions are enforced. Similarly, the boundary of each fracture $\partial F_{i}$, $i=1, \ldots, N_{F}$, is split in a Dirichlet and Neumann part, $\gamma_{i D}$ and $\gamma_{i N}$, respectively. If fracture $F_{i}$ lies in the interior of $\mathcal{D}$, then we set $\gamma_{i D}=\emptyset$, and homogeneous Neumann boundary conditions are prescribed on $\gamma_{i N} \equiv \partial F_{i}$. If $N_{F}=$ 1 , we assume that $\left|\gamma_{1 D}\right|>0$, whereas, if there is more than one fracture in the network, we allow $\gamma_{i D}=\emptyset$ for $i=1, \ldots, N_{F}$. The problem of the equilibrium distribution of the hydraulic head in $\mathcal{D}$ can be then stated in strong formulation as:

$$
\begin{aligned}
-\nabla \cdot\left(\mathbf{K}_{\mathcal{D}} \nabla H_{\mathcal{D}}\right) & =f \quad \text { in } \tilde{\mathcal{D}} \\
-\nabla_{i} \cdot\left(\mathbf{K}_{i} \nabla_{i} H_{i}\right) & =-\left[\mathbf{K}_{\mathcal{D}} \nabla H_{\mathcal{D}} \cdot \mathbf{n}\right]_{\Gamma_{i}} \quad \text { in } \tilde{F}_{i}, \\
i & =1, \ldots, N_{F} \\
\left(H_{\mathcal{D}}\right)_{\mid \Gamma_{i}^{ \pm}} & =H_{i}, \quad i=1, \ldots, N_{F} \\
H_{i} & =H_{j}, \quad \text { on } S_{m}, m=1, \ldots, N_{S}, \\
i, j & =\sigma(m)
\end{aligned}
$$

$\left[\mathbf{K}_{i} \nabla_{i} H_{i} \cdot \mathbf{n}\right]_{S_{m}}=-\left[\mathbf{K}_{j} \nabla_{j} H_{j} \cdot \mathbf{n}\right]_{S_{m}}, m=1, \ldots, N_{S}$,

$$
\begin{aligned}
& i, j=\sigma(m) \\
& H_{\mathcal{D}}=0 \quad \text { on } \Gamma_{D}
\end{aligned}
$$

$\mathbf{K}_{\mathcal{D}} \nabla H_{\mathcal{D}} \cdot \mathbf{n}_{\Gamma_{N}}=0 \quad$ on $\Gamma_{N}$

$$
H_{i}=0 \quad \text { on } \gamma_{i D}, i=1, \ldots, N_{F}
$$

$\mathbf{K}_{i} \nabla_{i} H_{i} \cdot \mathbf{n}_{\gamma_{i N}}=0 \quad$ on $\gamma_{i N}, i=1, \ldots, N_{F}$ where $H_{\mathcal{D}}$ is the hydraulic head in $\tilde{\mathcal{D}}, H_{i}$ the hydraulic head on $F_{i}, i=1, \ldots, N_{F}$ and $f$ is a volumetric source term. The operator $\nabla$ represents the three-dimensional gradient in $\tilde{\mathcal{D}}, \nabla_{i}$ is the two-dimensional gradient on the plane containing fracture $F_{i}$, whereas $\mathbf{K}_{\mathcal{D}}(\mathbf{x}) \in \mathbb{R}^{3 \times 3}$, for $\mathbf{x} \subset$ $\tilde{\mathcal{D}}$ is a symmetric positive definite matrix representing the transmissivity of the porous matrix and $\mathbf{K}_{i}(\mathbf{x}) \in \mathbb{R}^{2 \times 2}, \mathbf{x} \subset$ $F_{i}$ is a symmetric positive definite matrix representing the tangential transmissivity of the fracture $F_{i}$ on its tangential plane. Finally, $\mathbf{n}_{\Gamma_{N}}$ is the outward unit normal vector to $\Gamma_{N}$, and for a given index $i=1, \ldots, N_{F}, \mathbf{n}_{\gamma_{i N}}$ the outward unit normal vector $\gamma_{i N}$ on the plane of fracture $F_{i}$.

Here, for simplicity, we have considered only the source term on the fractures deriving from the exchange with the porous matrix and homogeneous boundary conditions, but the extension to a more general case is immediate. Conditions Eq. 3 and Eq. 4 express the continuity of the solution at fracture-matrix interfaces and at fracture intersections, respectively, whereas Eq. 5 enforces the balance of fluxes at the traces.

Let us now introduce the following functional spaces: first, on each fracture $F_{i}, i=1, \ldots, N_{F}$, we define the function space $\mathrm{V}_{i}$ as $\mathrm{V}_{i}=\mathrm{H}_{D}^{1}\left(F_{i}\right)=$ $\left\{v \in \mathrm{H}^{1}\left(F_{i}\right): v_{\mid \gamma_{i D}=0}\right\}$; then on the whole three dimensional domain $\mathcal{D}$, the space $\mathrm{H}_{\Omega}^{1}(\mathcal{D})$ is defined as the space of functions in $\mathrm{H}_{0}^{1}(\mathcal{D})$ whose trace on each interface $\Gamma_{i}^{ \pm}$ $i=1, \ldots, N_{F}$ is a function in $\mathrm{V}_{i}$, i.e.:

$\mathrm{H}_{\Omega}^{1}(\mathcal{D})=\left\{v \in \mathrm{H}_{0}^{1}(\mathcal{D}): v_{\mid \Gamma_{D}=0}, v_{\mid \Gamma_{i}^{ \pm}} \in \mathrm{V}_{i}, i=1, \ldots, N_{F}\right\}$.

Also, on each trace $S_{m}, m=1, \ldots, N_{S}$ we set the spaces $\mathcal{U}^{m}=\mathrm{H}^{-\frac{1}{2}}\left(S_{m}\right)$ and $\mathcal{H}^{m}=\mathrm{H}^{\frac{1}{2}}\left(S_{m}\right)$. We introduce the following variables: $U_{i}^{m} \in \mathcal{U}^{m}$ defined on trace $S_{m}$ of fracture $F_{i}$ as

$$
\begin{aligned}
U_{i}^{m} & =\left[\mathbf{K}_{i} \nabla_{i} H_{i} \cdot \mathbf{n}\right]_{S_{m}}+\alpha H_{i \mid S_{m}}, \\
\forall i & =1, \ldots, N_{F}, \forall m \in \mathcal{S}_{i},
\end{aligned}
$$

thus representing a sort of internal Robin boundary condition on the traces; and, for all $i=1, \ldots, N_{F}, Q_{i} \in V_{i}^{\prime}$, with

$$
Q_{i}:=\left[\mathbf{K}_{\mathcal{D}} \nabla H_{\mathcal{D}} \cdot \mathbf{n}\right]_{\Gamma_{i}}+\beta H_{\mathcal{D} \mid \Gamma_{i}},
$$

thus again being a linear combination of the jump of the conormal derivative of $H_{\mathcal{D}}$ across interface $\Gamma_{i}$ and the trace of $H_{\mathcal{D}}$ on $\Gamma_{i}$, and $\mathrm{V}_{i}^{\prime}$ the dual of $\mathrm{V}_{i}$. We remark that, as $H_{\mathcal{D}} \in \mathrm{H}_{\Omega}^{1}(\mathcal{D})$ the hydraulic head is continuous across interfaces $\Gamma_{i} \equiv F_{i} \subset \mathcal{D}$.

We also define the bilinear forms: $a_{\mathcal{D}}: \mathrm{H}_{\Omega}^{1}(\mathcal{D}) \times$ $\mathrm{H}_{\Omega}^{1}(\mathcal{D}) \mapsto \mathbb{R}$,

$$
a_{\mathcal{D}}(v, w)=\int_{\mathcal{D}} \mathbf{K}_{\mathcal{D}} \nabla v \nabla w \mathrm{~d} \mathcal{D}+\beta \sum_{i=1}^{N_{F}} \int_{\Gamma_{i}} v_{\mid \Gamma_{i}} w_{\mid \Gamma_{i}} \mathrm{~d} \Gamma ;
$$


for all $i=1, \ldots, N_{F}$, bilinear forms $a_{F_{i}}: \mathrm{V}_{i} \times \mathrm{V}_{i} \mapsto \mathbb{R}$,

$$
\begin{aligned}
a_{F_{i}}\left(v_{i}, w_{i}\right)= & \int_{F_{i}} \mathbf{K}_{i} \nabla_{i} v_{i} \nabla_{i} w_{i} \mathrm{~d} F_{i} \\
& +\alpha \sum_{m \in \mathcal{S}_{i}} \int_{S_{m}} v_{i \mid S_{m}} w_{i \mid S_{m}} \mathrm{~d} S ;
\end{aligned}
$$

$b_{i}: \mathrm{V}_{i}^{\prime} \times \mathrm{V}_{i} \mapsto \mathbb{R}$

$b_{i}(q, v)=\langle q, v\rangle_{\mathrm{V}_{i}^{\prime}, \mathrm{V}_{i}}$

and, for $m=1, \ldots, N_{S}$, form $c^{m}: \mathcal{U}^{m} \times \mathcal{H}^{m} \mapsto \mathbb{R}$,

$c^{m}\left(u^{m}, v\right)=\left\langle u^{m}, v\right\rangle_{\mathcal{U}^{m}, \mathcal{H}^{m}}$.

Then, problem Eq. 1-Eq. 9 can be written in weak formulation as: find $H_{\mathcal{D}} \in \mathrm{H}_{\Omega}^{1}(\mathcal{D}), H_{i} \in \mathrm{V}_{i}, Q_{i} \in \mathrm{V}_{i}^{\prime}$, $U_{i}^{m} \in \mathcal{U}_{i}^{m}, m \in \mathcal{S}_{i}$, such that, for all $v \in \mathrm{H}_{\Omega}^{1}(\mathcal{D})$, for all $w_{i} \in \mathrm{V}_{i}, i=1, \ldots, N_{F}$ :

$$
\begin{aligned}
& a_{\mathcal{D}}\left(H_{\mathcal{D}}, v\right)-\sum_{i=1}^{N_{F}} b_{i}\left(Q_{i}, v_{\mid \Gamma_{i}}\right)=\langle f, v\rangle_{\left(\mathrm{H}_{\Omega}^{1}\right)^{\prime}, \mathrm{H}_{\Omega}^{1}} \\
& a_{F_{i}}\left(H_{i}, w_{i}\right)-\beta\left(H_{\mathcal{D} \mid F_{i}}, w_{i}\right)_{F_{i}}-\sum_{m \in \mathcal{S}_{i}} c^{m}\left(U_{i}^{m}, w_{i \mid S_{m}}\right) \\
& =-b_{i}\left(Q_{i}, w_{i}\right),
\end{aligned}
$$

being $(v, w)_{\omega}$ the scalar product in $\mathrm{L}^{2}(\omega)$. The coupling conditions in weak form are given by: for all $i=1, \ldots, N_{F}$, and for all $m=1, \ldots, N_{S}$

$$
\begin{aligned}
b_{i}\left(H_{\mathcal{D} \mid F_{i}}-H_{i}, \mu_{i}\right) & =0, \quad \forall \mu_{i} \in \mathrm{V}_{i}^{\prime}, \\
c^{m}\left(\eta^{m}, H_{i \mid S_{m}}-H_{j \mid S_{m}}\right) & =0, \quad \forall \eta^{m} \in \mathcal{U}^{m}, \quad i, j=\sigma(m)
\end{aligned}
$$

$c^{m}\left(U_{i}^{m}+U_{j}^{m}-\alpha\left(H_{i \mid S_{m}}+H_{j \mid S_{m}}\right), v^{m}\right)=0, \quad \forall v^{m} \in \mathcal{H}^{m}, i, j=\sigma(m)$.

Parameters $\alpha>0$ and $\beta>0$ ensure stability of the problems written independently on each fracture and on the three dimensional domain. This is required to obtain a discrete formulation suitable for parallel computing. Moreover, the choice $\alpha>0$ and $\beta>0$ allows to have Dirichlet boundary conditions alternatively on the three dimensional domain or on the fracture network, i.e. we can have $\Gamma_{D}=\varnothing$ if $\gamma_{i D} \neq \varnothing$ for at least one fracture index $i=1, \ldots, N_{F}$, or $\gamma_{i D}=\emptyset$ for all the fractures if $\Gamma_{D} \neq \emptyset$.

Problem Eq. 12-Eq. 16 is well posed. To show this, let us introduce the function space $\mathrm{H}_{\Omega^{+}}^{1}(\mathcal{D})$ defined as:

$$
\begin{aligned}
& \mathrm{H}_{\Omega^{+}}^{1}(\mathcal{D})=\left\{v \in \mathrm{H}_{0}^{1}(\mathcal{D}): v_{i}:=v_{\mid F_{i}} \in \mathrm{V}_{i},\right. \\
& \forall i=1, \ldots, N_{F}, v_{i \mid S_{m}}=v_{j \mid S_{m}}, \\
& \left.\forall m=1, \ldots, N_{S}, i, j=\sigma(m)\right\}
\end{aligned}
$$

and thus incorporating the matching conditions at the interfaces. Let us then write the following problem: find
$H \in \mathrm{H}_{\Omega^{+}}^{1}(\mathcal{D})$ such that, for all $v \in \mathrm{H}_{\Omega^{+}}^{1}(\mathcal{D})$

$(\mathbf{K} \nabla H, \nabla v)_{\mathcal{D}}+\sum_{i=1}^{N_{F}}\left(\mathbf{K}_{i} \nabla_{i} H_{i}, \nabla_{i} v_{i}\right)_{F_{i}}=\langle q, v\rangle$.

Problem Eq. 17 is well posed, as it can be easily seen that $\mathrm{H}_{\Omega^{+}}^{1}(\mathcal{D})$ is an Hilbert space with the scalar product, [12]:

$$
(v, w)_{\mathrm{H}_{\Omega^{+}}^{1}}:=(\mathbf{K} \nabla v, \nabla w)_{\tilde{\mathcal{D}}}+\sum_{i=1}^{N_{F}}\left(\mathbf{K}_{i} \nabla_{i} w_{i}, \nabla_{i} v_{i}\right)_{F_{i}} .
$$

Problem Eq. 12-Eq. 16 is equivalent to problem Eq. 17; indeed, recalling that, for $v \in \mathrm{H}_{\Omega^{+}}^{1}(\mathcal{D})$, conditions Eq. 14Eq. 15 are satisfied by construction. Moreover summing Eq. 13 for $i=1, \ldots, N_{F}$ and Eq. 12, using Eq. 16 and the definition of $U_{i}^{m}$ and $Q_{i}$, for $i=1, \ldots, N_{F}, m \in \mathcal{S}_{i}$, we get Eq. 17. We propose a reformulation of problem Eq. 12Eq. 16 well suited for discretization on non conforming meshes and parallel computing, based on a PDE constrained optimization approach. To this end, we introduce a cost functional expressing the error in the fulfilment of the interface conditions as continuity and flux conservation:

$$
\begin{gathered}
J\left(H_{\mathcal{D}}, H_{\mathcal{F}}, U_{\mathcal{S}}\right):=\sum_{i=1}^{N_{F}}\left(\left\|H_{\mathcal{D} \mid F_{i}}-H_{i}\right\|_{\mathrm{V}_{i}}^{2}\right) \\
+\sum_{m=1}^{N_{S}}\left(\left\|H_{i \mid S_{m}}-H_{j \mid S_{m}}\right\|_{\mathcal{H}^{m}}^{2}+\| U_{i}^{m}+U_{j}^{m}\right. \\
\left.-\alpha\left(H_{i \mid S_{m}}+H_{j \mid S_{m}}\right) \|_{\mathcal{U}^{m}}^{2}\right),
\end{gathered}
$$

being $H_{\mathcal{F}}:=\prod_{i=1}^{N_{F}} H_{i}$ and $U_{\mathcal{S}}=\prod_{m=1}^{N_{S}} \prod_{i \in \sigma(m)} U_{i}^{m}$. Setting, finally, $Q_{\mathcal{F}}:=\prod_{i=1}^{N_{F}} Q_{i}$, the solution to problem Eq. 12-Eq. 16 is obtained as the minimum of functional $J\left(H_{\mathcal{D}}, H_{\mathcal{F}}, U_{\mathcal{S}}\right)$ constrained by the PDE equations on the 3D domain and on the fractures:

$$
\begin{aligned}
\min _{Q_{\mathcal{F}}, U_{\mathcal{S}}} J\left(H_{\mathcal{D}}, H_{\mathcal{F}}, U_{\mathcal{S}}\right) \\
\text { constrained by Eq. } 12 \\
\text { and by Eq. } 13 \forall i=1, \ldots, N_{F} .
\end{aligned}
$$

\section{Discrete formulation}

The PDE constrained optimization formulation is specifically designed to allow for an easy discretization of the problem using non conforming meshes and to obtain a discrete problem suitable for effective resolution using parallel computing resources. The imposition of the interface constraints expressed by Eqs. 14- 16 with a standard approach requires some sort of mesh conformity at the interfaces: either a perfect matching of the nodes on the meshes to enforce conditions by means of degrees of freedom equality constraints, or the weaker condition of alignment of mesh 
edges with the interfaces, to use mortaring techniques. In contrast, the imposition of interface conditions through the functional only requires the computation of integrals on the traces, as shown below, and thus meshes can be arbitrarily placed with respect to the interfaces, see Figure 2 for an example of non-conforming meshes in the rock matrix and on the fractures. Further, the minimization process allows to decouple the problems on the fractures and on the three-dimensional domain, for parallel computing.

The discretization strategy proposed in this work is based on the use of standard finite elements on tetrahedra for the three dimensional domain and finite elements on triangles for the fractures. Let us then denote by $\mathcal{T}_{\delta_{\mathcal{D}}}^{\mathcal{D}}$ the tetrahedral mesh on $\mathcal{D}$, characterized by a mesh parameter $\delta_{\mathcal{D}}$, by $\mathcal{T}_{\delta_{F_{i}}}^{i}$ a triangular mesh on $F_{i}, i=1, \ldots, N_{F}$, with mesh parameter $\delta_{F_{i}}$, and by $\mathcal{T}_{\delta_{\Gamma_{i}}}^{i}$ a possibly different triangular mesh on $F_{i}$, with mesh parameter $\delta_{\Gamma_{i}}$. We further introduce a discretization of the one-dimensional traces, different on each fracture, denoted by $\mathcal{T}_{\delta_{S_{m}, i}}^{i}$, with mesh parameter $\delta_{S_{m}, i}, i=1, \ldots, N_{F}, m \in \mathcal{S}_{i}^{m}$. We denote by $h_{\mathcal{D}}$ the finite dimensional approximation of $H_{\mathcal{D}}$ on $\mathcal{T}_{\delta_{\mathcal{D}}}^{\mathcal{D}}, h_{\mathcal{D}}=$ $\sum_{k=1}^{\mathcal{N}_{h_{\mathcal{D}}}} h_{\mathcal{D}, k} \phi_{k}$, with $\mathcal{N}_{h_{\mathcal{D}}}$ the number of degrees of freedom (dofs) and $\phi_{k}$ a finite element basis function in 3D; for $i=1, \ldots, N_{F}$, we further call $h_{i}$ the approximation of $H_{i}$ on $\mathcal{T}_{\delta_{F_{i}}}^{i}, h_{i}=\sum_{k=1}^{\mathcal{N}_{h_{i}}} h_{i, k} \psi_{i, k}$, with $\mathcal{N}_{h_{i}}$ the number of dofs and $\psi_{i, k}$ a $2 \mathrm{D}$ basis function; $q_{i}$ the approximation of $Q_{i}$ on $\mathcal{T}_{\delta_{\Gamma_{i}}}^{i}, q_{i}=\sum_{k=1}^{\mathcal{N}_{q_{i}}} q_{i, k} \varphi_{i, k}$ having $\mathcal{N}_{q_{i}}$ dofs, and $\varphi_{i, k}$ one basis function; $u_{i}^{m}$ the approximation of $U_{i}^{m}$ on $\mathcal{T}_{\delta_{S_{m}, i}}^{i}, u_{i}^{m}=$ $\sum_{k=1}^{\mathcal{N}_{u_{i}}^{m}} u_{i, k}^{m} \varrho_{i, k}^{m}$, with $\mathcal{N}_{u_{i}}^{m}$ dofs and $\varrho_{i, k}^{m}$ a basis function. Tables 1-2 summarize the labels used for the dimensions of the discrete variables, the name used to denote the basis functions and the notation used, in the following, for the matrices collecting integrals of these basis functions. We build arrays of dofs by collecting column-wise the dofs of each discrete function and with abuse of notation we denote the dof array with the same symbol of the corresponding
Table 1 Labels used for the dimension of discrete variables

\begin{tabular}{lll}
\hline Label & Description & Definition \\
\hline $\mathcal{N}_{h_{\mathcal{D}}}$ & Number of dofs for $h_{\mathcal{D}}$ & \\
$\mathcal{N}_{h_{i}}$ & Number of dofs for $h_{i}$ on $\mathcal{T}_{\delta_{F_{i}}}^{i}$ & \\
$\mathcal{N}_{q_{i}}$ & Number of dofs for $q_{i}$ on $\mathcal{T}_{\delta_{\Gamma_{i}}^{i}}^{i}$ & \\
$\mathcal{N}_{u_{i}}^{m}$ & Number of dofs for $u_{i}^{m}$ on $\mathcal{T}_{\delta_{S_{m}, i}}^{i}$ & \\
$\mathcal{N}_{h_{\mathcal{F}}}$ & Number of dofs for $h_{\mathcal{F}}$ & $\sum_{i=1}^{N_{F}} \mathcal{N}_{h_{i}}$ \\
$\mathcal{N}_{q}$ & Number of dofs for $q$ & $\sum_{i=1}^{N_{F}} \mathcal{N}_{q_{i}}$ \\
$\mathcal{N}_{u_{i}}$ & Number of dofs for $u_{i}$ & $\sum_{m \in \mathcal{S}_{i}} \mathcal{N}_{u_{i}}^{m}$ \\
$\mathcal{N}_{u_{i}}^{+}$ & Number of dofs for $u^{+}$ & $\sum_{m \in \mathcal{S}_{i}} \sum_{k \in \sigma(m)} \mathcal{N}_{u_{i}}^{m}$ \\
$\mathcal{N}_{u}$ & Number of dofs for $u$ & $\sum_{m=1}^{N_{S}} \sum_{k \in \sigma(m)} \mathcal{N}_{u_{i}}^{m}$ \\
$\mathcal{N}_{h}$ & Number of dofs for $h$ & $\mathcal{N}_{h_{\mathcal{F}}}+\mathcal{N}_{h_{\mathcal{D}}}$ \\
\hline
\end{tabular}

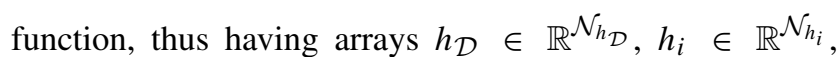

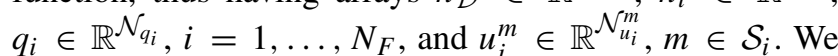
define arrays $u^{m}, m=1, \ldots, N_{S}$, as $u^{m}=\left[\left(u_{i}^{m}\right)^{T}\left(u_{j}^{m}\right)^{T}\right]^{T}$ for $i, j=\sigma(m)$ with $i<j$, and we further collect column wise arrays $h_{i}, q_{i}, u_{i}^{m}$ and $u^{m}$ forming:

$$
\begin{aligned}
& h_{\mathcal{F}}=\left[\begin{array}{c}
h_{1} \\
\vdots \\
h_{N_{F}}
\end{array}\right], q=\left[\begin{array}{c}
q_{1} \\
\vdots \\
q_{N_{F}}
\end{array}\right], u_{i}=\left[\begin{array}{c}
u_{i}^{m_{1}} \\
\vdots \\
u_{i}^{m_{\sharp S_{i}}}
\end{array}\right] \text {, } \\
& u_{i}^{+}=\left[\begin{array}{c}
u^{m_{1}} \\
\vdots \\
u^{m_{\sharp \mathcal{S}_{i}}}
\end{array}\right], u=\left[\begin{array}{c}
u^{1} \\
\vdots \\
u^{m} \\
\vdots \\
u^{N_{S}}
\end{array}\right] \text {, }
\end{aligned}
$$

where $m_{1}, \ldots, m_{\sharp \mathcal{S}_{i}}$ are the indexes in $\mathcal{S}_{i}$ ordered increasingly.
Fig. 2 Polygonal tessellation on a sample fracture given by the intersection of a tetrahedral mesh with the fracture plane (blue in the right panel) overlapped with the fracture triangular mesh (red in the right panel)

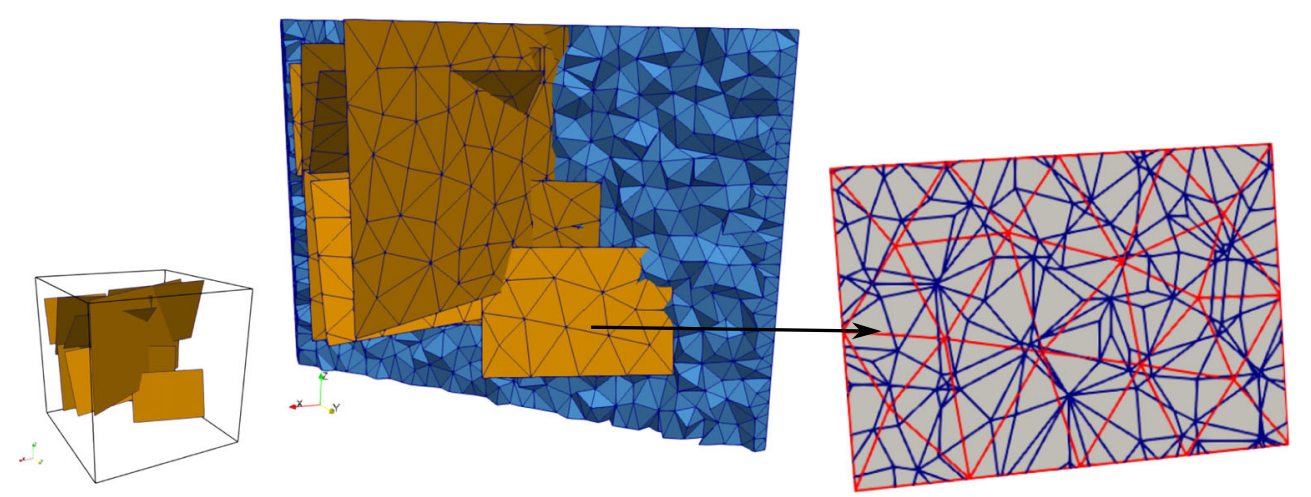


Table 2 Summary of nomenclature used for the discrete matrices: involved discrete function names and basis functions

\begin{tabular}{llll}
\hline Matrix letter & function(s) & basis functions & Integration domain \\
\hline $\mathbf{A}_{\mathcal{D}}$ & $h_{\mathcal{D}}$ & $\phi$ & $\mathcal{D}$ \\
$\mathbf{A}_{i}$ & $h_{i}$ & $\psi_{i}$ & $F_{i}$ \\
$\mathbf{B}_{i j}^{m}$ & $h_{i}, u_{i}^{m}$ & $\psi_{i}, \varrho_{j}^{m}$ & $S_{m}$ \\
$\mathbf{C}_{i j}^{m}$ & $u_{i}^{m}, u_{j}^{m}$ & $\varrho_{i}^{m}, \varrho_{j}^{m}$ & $S_{m}$ \\
$\mathbf{D}_{i}$ & $h_{i}, q$ & $\psi_{i}, \varphi_{i}$ & $F_{i}$ \\
$\mathbf{E}_{i}$ & $h_{\mathcal{D}}, q$ & $\phi, \varphi_{i}$ & $F_{i}$ \\
$\mathbf{G}_{\mathcal{D}}^{i}$ & $h_{\mathcal{D}}$ & $\phi$ & $F_{i}$ \\
$\mathbf{G}_{F}^{i}$ & $h_{i}$ & $\psi_{i}$ & $F_{i}$ \\
$\mathbf{G}_{\mathcal{D} F}^{i}$ & $h_{\mathcal{D}}, h_{i}$ & $\phi, \psi_{i}$ & $F_{i}$ \\
$\mathbf{G}_{i j}^{m}$ & $h_{i}, h_{j}$ & $\psi_{i}, \psi_{j}$ & $S_{m}$ \\
\hline
\end{tabular}

The discrete version of functional $J$ is the following:

$$
\begin{gathered}
\mathcal{J}\left(h_{\mathcal{D}}, h_{\mathcal{F}}, u\right)=\sum_{i=1}^{N_{F}}\left\|h_{\mathcal{D} \mid F_{i}}-h_{i}\right\|_{\mathrm{L}^{2}\left(F_{i}\right)}^{2} \\
+\sum_{m=1}^{N_{S}}\left(\left\|h_{i}-h_{j}\right\|_{\mathrm{L}^{2}\left(S_{m}\right)}^{2}+\| u_{i}^{m}+u_{j}^{m}\right. \\
\left.-\alpha\left(h_{i}+h_{j}\right) \|_{\mathrm{L}^{2}\left(S_{m}\right)}^{2}\right)
\end{gathered}
$$

obtained replacing the discretized variables and using $\mathrm{L}^{2}$ norms. The discrete functional can be written in matrix form, computing the integrals of the basis functions and collecting the values into matrices. Considering the first norm in $\mathcal{J}$, we have:

$$
\begin{aligned}
& \left\|h_{\mathcal{D} \mid F_{i}}-h_{i}\right\|_{\mathrm{L}^{2}\left(F_{i}\right)}^{2} \\
& =\int_{F_{i}}\left(\sum_{k=1}^{\mathcal{N}_{h_{\mathcal{D}}}} h_{\mathcal{D}, k} \phi_{k \mid F_{i}}-\sum_{j=1}^{\mathcal{N}_{h_{i}}} h_{i, j} \psi_{i, j}\right)^{2} \mathrm{~d} F_{i}
\end{aligned}
$$

and we can define three matrices as follows, for each $i=$ $1, \ldots, N_{F}, \mathbf{G}_{\mathcal{D}}^{i} \in \mathbb{R}^{\mathcal{N}_{h_{\mathcal{D}}} \times \mathcal{N}_{h_{\mathcal{D}}}}, \mathbf{G}_{\mathcal{D} F}^{i} \in \mathbb{R}^{\mathcal{N}_{h_{\mathcal{D}}} \times \mathcal{N}_{h_{i}}}, \mathbf{G}_{F}^{i} \in$ $\mathbb{R}^{\mathcal{N}_{h_{i}} \times \mathcal{N}_{h_{i}}}$ :

$$
\begin{aligned}
\left(\mathbf{G}_{\mathcal{D}}^{i}\right)_{k, \ell} & =\int_{F_{i}} \phi_{k \mid F_{i}} \phi_{k \mid F_{i}}, \quad\left(\mathbf{G}_{\mathcal{D} F}^{i}\right)_{k, \ell}=\int_{F_{i}} \phi_{k \mid F_{i}} \psi_{i, \ell}, \\
\left(\mathbf{G}_{F}^{i}\right)_{k, \ell} & =\int_{F_{i}} \psi_{i, k} \psi_{i, \ell}
\end{aligned}
$$

such that

$$
\left\|h_{\mathcal{D} \mid F_{i}}-h_{i}\right\|_{\mathrm{L}^{2}\left(F_{i}\right)}^{2}=\left[h_{\mathcal{D}}^{T} h_{i}^{T}\right]\left[\begin{array}{cc}
\mathbf{G}_{\mathcal{D}}^{i} & -\mathbf{G}_{\mathcal{D} F}^{i} \\
-\left(\mathbf{G}_{\mathcal{D} F}^{i}\right)^{T} & \mathbf{G}_{F}^{i}
\end{array}\right]\left[\begin{array}{c}
h_{\mathcal{D}} \\
h_{i}
\end{array}\right] .
$$

The computation of matrix $\mathbf{G}_{\mathcal{D} F}^{i}$ is not straightforward, as the two involved variables are defined on different meshes. In particular, the intersection of the three dimensional tetrahedral mesh with the fracture plane needs to be computed. This operation defines a polygonal tessellation of $F_{i}$ which is then sub-triangulated, thus generating a triangular interface mesh. This sub-triangulation process can be performed without any mesh quality requirement, as the resulting mesh is used only for quadrature purposes. The computation of the elements in $\mathbf{G}_{\mathcal{D F}}^{i}$ is finally performed first computing the intersection of the elements of the interface mesh with the elements in $\mathcal{T}_{\delta_{F}}^{i}$, and subsequently the required integral on the intersection region. Element neighbourhood information is used to efficiently perform the task. The computation of the interface mesh is a quite complex and expensive task. Also in this case element neighbourhood information is used for efficiency, and further can be performed independently fracture by fracture and thus in parallel, which is of paramount importance for the applicability of the method to complex geometries.

We can proceed similarly with the remaining terms of the functional $\mathcal{J}$; to this end, for $m=1, \ldots, N_{S}$, and all the possible couples of indexes $i, j$ such that $i, j=\sigma(m)$, we define matrices $\mathbf{G}_{i j}^{m} \in \mathbb{R}^{\mathcal{N}_{h_{i}} \times \mathcal{N}_{F_{j}}}, \mathbf{B}_{i j}^{m} \in \mathbb{R}^{\mathcal{N}_{h_{i}} \times \mathcal{N}_{j}^{m}}$, $\mathbf{C}_{i j}^{m} \in \mathbb{R}^{\mathcal{N}_{u_{i}}^{m} \times \mathcal{N}_{j}^{m}}:$

$$
\begin{aligned}
\left(\mathbf{G}_{i j}^{m}\right)_{k, \ell} & =\int_{S_{m}} \psi_{i, k \mid S_{m}} \psi_{j, \ell \mid S_{m}}, \\
\left(\mathbf{B}_{i j}^{m}\right)_{k, \ell} & =\int_{S_{m}} \psi_{i, k \mid S_{m}} \varrho_{j, \ell}^{m}, \quad\left(\mathbf{C}_{i j}^{m}\right)_{k, \ell}=\int_{S_{m}} \varrho_{i, k}^{m} \varrho_{j, \ell}^{m}
\end{aligned}
$$

such that

$$
\left\|h_{i}-h_{j}\right\|_{\mathrm{L}^{2}\left(S_{m}\right)}^{2}=\left[\begin{array}{ll}
h_{i}^{T} & h_{j}^{T}
\end{array}\right]\left[\begin{array}{cc}
\mathbf{G}_{i i}^{m} & -\mathbf{G}_{i j}^{m} \\
-\mathbf{G}_{j i}^{m} & \mathbf{G}_{j j}^{m}
\end{array}\right]\left[\begin{array}{l}
h_{i} \\
h_{j}
\end{array}\right],
$$

and

$$
\begin{aligned}
& \left\|u_{i}^{m}+u_{j}^{m}-\alpha\left(h_{i}+h_{j}\right)\right\|_{\mathrm{L}^{2}\left(S_{m}\right)}^{2} \\
& =\left(\left[\left(u_{i}^{m}\right)^{T}\left(u_{j}^{m}\right)^{T}\right]\left[\begin{array}{ll}
\mathbf{C}_{i i}^{m} & \mathbf{C}_{i j}^{m} \\
\mathbf{C}_{j i}^{m} & \mathbf{C}_{j j}^{m}
\end{array}\right]\right. \\
& \left.-2 \alpha\left[\begin{array}{ll}
h_{i}^{T} & h_{j}^{T}
\end{array}\right]\left[\begin{array}{ll}
\mathbf{B}_{i i}^{m} & \mathbf{B}_{i j}^{m} \\
\mathbf{B}_{j i}^{m} & \mathbf{B}_{j j}^{m}
\end{array}\right]\right)\left[\begin{array}{c}
u_{i}^{m} \\
u_{j}^{m}
\end{array}\right] \\
& +\alpha^{2}\left[\begin{array}{ll}
h_{i}^{T} & h_{j}^{T}
\end{array}\right]\left[\begin{array}{ll}
\mathbf{G}_{i i}^{m} & \mathbf{G}_{i j}^{m} \\
\mathbf{G}_{j i}^{m} & \mathbf{G}_{j j}^{m}
\end{array}\right]\left[\begin{array}{l}
h_{i} \\
h_{j}
\end{array}\right] .
\end{aligned}
$$

We can collect these matrices, defined locally at the various interfaces into global matrices to derive a compact form of the functional. Let us define matrix $\mathbf{G}^{\mathcal{S}} \in$

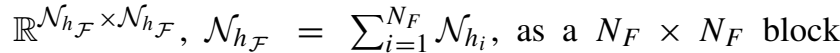
matrix, with diagonal blocks in positions $i-i$ are given by $\left(1+\alpha^{2}\right) \sum_{m \in \mathcal{S}_{i}} \mathbf{G}_{i i}^{m}, i=1, \ldots, N_{F}$. Extra diagonal blocks in positions $i-j(i \neq j)$ are instead equal to $\left(\alpha^{2}-1\right) \mathbf{G}_{i j}^{m}$, if $i, j=\sigma(m)$, or a zero block otherwise. Further let us define matrix $\mathbf{G}_{\mathcal{D}}^{\mathcal{F}}=\sum_{i=1}^{N_{F}} \mathbf{G}_{\mathcal{D}}^{i}$, and matrices $\mathbf{G}_{\mathcal{D} F}^{\mathcal{F}} \in \mathbb{R}^{\mathcal{N}_{h_{\mathcal{D}}} \times \mathcal{N}_{h_{\mathcal{F}}}}$ and $\mathbf{G}_{F}^{\mathcal{F}} \in \mathbb{R}^{\mathcal{N}_{h_{\mathcal{F}}} \times \mathcal{N}_{h_{\mathcal{F}}}}$ respectively as

$$
\begin{aligned}
& \mathbf{G}_{\mathcal{D} F}^{\mathcal{F}}=\left[\mathbf{G}_{\mathcal{D} F}^{1} \cdots \mathbf{G}_{\mathcal{D} F}^{i} \cdots \mathbf{G}_{\mathcal{D} F}^{N_{F}}\right], \\
& \mathbf{G}_{F}^{\mathcal{F}}=\operatorname{diag}\left(\mathbf{G}_{F}^{1}, \ldots, \mathbf{G}_{F}^{i}, \ldots, \mathbf{G}_{F}^{N_{F}}\right)
\end{aligned}
$$


Matrix $\mathbf{G} \in \mathbb{R}^{\mathcal{N}_{h} \times \mathcal{N}_{h}}, \mathcal{N}_{h}=\mathcal{N}_{h_{\mathcal{F}}}+\mathcal{N}_{h_{\mathcal{D}}}$, is finally set as $\mathbf{G}=\left[\begin{array}{cc}\mathbf{G}_{\mathcal{D}}^{\mathcal{F}} & \mathbf{G}_{\mathcal{D} F}^{\mathcal{F}} \\ \left(\mathbf{G}_{\mathcal{D} F}^{\mathcal{F}}\right)^{T} & \mathbf{G}_{F}^{\mathcal{F}}+\mathbf{G}^{\mathcal{S}}\end{array}\right]$

For all $i=1, \ldots, N_{F}$, let us assemble matrices $\mathbf{B}_{i}^{+} \in$

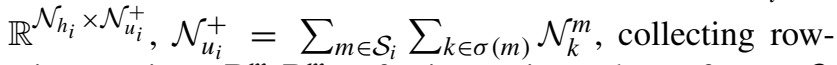
wise matrices $\left[\mathbf{B}_{i j}^{m} \mathbf{B}_{i k}^{m}\right]$, for increasing values of $m \in \mathcal{S}_{i}$ and $j, k=\sigma(m), j<k$, i.e.:

$\mathbf{B}_{i}^{+}=\left[\begin{array}{lllll}\mathbf{B}_{i j}^{m_{1}} & \mathbf{B}_{i k}^{m_{1}} & \cdots & \mathbf{B}_{i p}^{m_{\sharp \mathcal{S}_{i}}} & \mathbf{B}_{i z}^{m_{\sharp \mathcal{S}_{i}}}\end{array}\right]$

with $p, z=\sigma\left(m_{\sharp \mathcal{S}_{i}}\right), p<z$. Let us introduce matrices $\mathbf{R}_{i}^{+} \in \mathbb{R}^{\mathcal{N}_{u_{i}}^{+} \times \mathcal{N}_{u}}$, with $\mathcal{N}_{u}=\sum_{m=1}^{N_{S}} \sum_{k \in \sigma(m)} \mathcal{N}_{k}^{m}$, defined such that $u_{i}^{+}=\mathbf{R}_{i}^{+} u$. Matrix $\mathbf{B}^{+} \in \mathbb{R}^{\mathcal{N}_{h_{\mathcal{F}}} \times \mathcal{N}_{u}}$ is finally obtained collecting column wise matrices $\mathbf{B}_{i}^{+} \mathbf{R}_{i}^{+}$. Matrix $\mathbf{C} \in \mathbb{R}^{\mathcal{N}_{u} \times \mathcal{N}_{u}}$, is a block diagonal matrix with $N_{S} \times N_{S}$ diagonal blocks, each diagonal block in position $m-m$ being equal to

$$
\left[\begin{array}{ll}
\mathbf{C}_{i i}^{m} & \mathbf{C}_{i j}^{m} \\
\mathbf{C}_{j i}^{m} & \mathbf{C}_{j j}^{m}
\end{array}\right], \quad i, j=\sigma(m), i<j
$$

The matrix formulation of $\mathcal{J}$ then reads as:

$\mathcal{J}=\left[\begin{array}{ll}h_{\mathcal{D}}^{T} & h_{\mathcal{F}}^{T}\end{array}\right] \mathbf{G}\left[\begin{array}{l}h_{\mathcal{D}} \\ h_{\mathcal{F}}\end{array}\right]+u^{T} \mathbf{C} u-\alpha h_{\mathcal{F}}^{T} \mathbf{B}^{+} u-\alpha u^{T} \mathbf{B}^{+T} h_{\mathcal{F}}$.

We can re-write also the discrete constraint equations in matrix form. We follow a standard procedure and we define $\operatorname{matrix} \mathbf{A}_{\mathcal{D}} \in \mathbb{R}^{\mathcal{N}_{h_{\mathcal{D}}} \times \mathcal{N}_{h_{\mathcal{D}}} \text { as }}$

$\left(\mathbf{A}_{\mathcal{D}}\right)_{k \ell}=\int_{\mathcal{D}} \mathbf{K}_{\mathcal{D}} \nabla \phi_{k} \nabla \phi_{\ell}+\beta \sum_{i=1}^{N_{F}} \int_{F_{i}} \phi_{k \mid F_{i}} \phi_{\ell \mid F_{i}}$,

where the integral on $F_{i}$ is performed on the interface mesh, generated by the intersection of the tetrahedral mesh with each fracture. Matrices $\mathbf{A}_{i} \in \mathbb{R}^{\mathcal{N}_{h_{\mathcal{F}}} \times \mathcal{N}_{h_{\mathcal{F}}}}$, for $i=$ $1, \ldots, N_{F}$ are defined by

$\left(\mathbf{A}_{i}\right)_{k \ell}=\int_{F_{i}} \mathbf{K}_{i} \nabla_{i} \psi_{i, k} \nabla_{i} \psi_{i, \ell}+\alpha \sum_{m \in \mathcal{S}_{i}} \int_{S_{m}} \psi_{i, k \mid S_{m}} \psi_{i, \ell \mid S_{m}}$

which form the diagonal blocks of block diagonal matrix $\mathbf{A}_{\mathcal{F}} \in \mathbb{R}^{\mathcal{N}_{h_{\mathcal{F}}} \times \mathcal{N}_{h_{\mathcal{F}}}, \mathbf{A}_{\mathcal{F}}}=\operatorname{diag}\left(\mathbf{A}_{1}, \ldots, \mathbf{A}_{N_{F}}\right)$. We introduce, for each fracture $F_{i}, i=1, \ldots, N_{F}$ matrices $\mathbf{R}_{i} \in \mathbb{R}^{\mathcal{N}_{u_{i}} \times \mathcal{N}_{u}}, \mathcal{N}_{u_{i}}=\sum_{m \in \mathcal{S}_{i}} \mathcal{N}_{u_{i}}^{m}$, defined such that $u_{i}=\mathbf{R}_{i} u$, and $\mathbf{B}_{i} \in \mathbb{R}^{\mathcal{N}_{h_{i}} \times \mathcal{N}_{u_{i}}}$ obtained collecting rowwise matrices $\mathbf{B}_{i i}^{m}$ for all $m \in \mathcal{S}_{i}$. These matrices are used for the definition of matrix $\mathbf{B} \in \mathbb{R}^{\mathcal{N}_{h_{\mathcal{F}}} \times \mathcal{N}_{u}}$, defined grouping column-wise matrices $\mathbf{B}_{i}$, for $i=1, \ldots, N_{F}$. Matrix $\mathbf{D} \in$

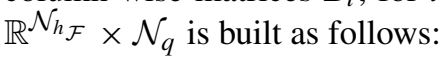

$\mathbf{D}=\left[\begin{array}{lll}\mathbf{D}_{1} & \cdots & \mathbf{D}_{N_{F}}\end{array}\right], \quad\left(\mathbf{D}_{i}\right)_{k \ell}=\int_{F_{i}} \psi_{i, k} \varphi_{i, \ell}$

with integrals computed on the intersection of the mesh $\mathcal{T}_{\delta_{\Gamma_{i}}}^{i}$ for variable $q_{i}$ with the mesh $\mathcal{T}_{\delta_{F_{i}}}^{i}$ for $h_{i}$. We finally introduce matrix $\mathbf{E} \in \mathbb{R}^{\mathcal{N}_{h_{\mathcal{D}}} \times \mathcal{N}_{q}}, \mathcal{N}_{q}=\sum_{i=1}^{N_{F}} \mathcal{N}_{q_{i}}$ as

$\mathbf{E}=\left[\begin{array}{lll}\mathbf{E}_{1} \cdots & \mathbf{E}_{N_{F}}\end{array}\right], \quad\left(\mathbf{E}_{i}\right)_{k \ell}=\int_{F_{i}} \phi_{k \mid F_{i}} \varphi_{i, \ell}$.

where integrals are computed intersecting the mesh $\mathcal{T}_{\delta_{\Gamma_{i}}}^{i}$ for variable $q_{i}$ with the triangulated interface mesh given by the intersection between the tetrahedral mesh with the fracture $F_{i}$.

Setting $h^{T}=\left[h_{\mathcal{D}}^{T} h_{\mathcal{F}}^{T}\right]$, the discrete formulation of the constrained minimization process is:

$$
\min \left(h^{T} \mathbf{G} h+u^{T} \mathbf{C} u-\alpha h_{\mathcal{F}}^{T} \mathbf{B}^{+} u-\alpha u^{T} \mathbf{B}^{+T} h_{\mathcal{F}}\right)
$$

constrained by

$$
\begin{aligned}
& \mathbf{A}_{\mathcal{D}} h_{\mathcal{D}}-\mathbf{E} q=b_{\mathcal{D}} \\
& \mathbf{A}_{\mathcal{F}} h_{\mathcal{F}}-\beta\left(\mathbf{G}_{\mathcal{D} F}^{\mathcal{F}}\right)^{T} h_{\mathcal{D}}-\mathbf{B} u+\mathbf{D} q=0
\end{aligned}
$$

being $b_{\mathcal{D}} \in \mathbb{R}^{\mathcal{N}_{h_{\mathcal{D}}}}$ the array resulting from the forcing term.

Let us now introduce the following matrices:

$$
\begin{aligned}
\mathcal{A} & =\left[\begin{array}{cc}
\mathbf{A}_{\mathcal{D}} & \mathbf{O} \\
-\beta\left(\mathbf{G}^{\mathcal{D} F}\right)^{T} & \mathbf{A}_{\mathcal{F}}
\end{array}\right], \mathcal{B}=\left[\begin{array}{cc}
\mathbf{E} & \mathbf{O} \\
-\mathbf{D} & \mathbf{B}
\end{array}\right], \\
\mathcal{B}^{+} & =\left[\begin{array}{cc}
\mathbf{O} & \mathbf{O} \\
\mathbf{O} & -\alpha \mathbf{B}^{+}
\end{array}\right], \mathcal{C}=\left[\begin{array}{ll}
\mathbf{O} & \mathbf{O} \\
\mathbf{O} & \mathbf{C}
\end{array}\right]
\end{aligned}
$$

and let us collect column-wise variables $q, u$ into variable $w$, then optimality conditions for problem Eq. 22-Eq. 24 are given by the following linear system:

$\mathcal{M}=\left[\begin{array}{ccc}\mathbf{G} & \mathcal{B}^{+} & \mathcal{A}^{T} \\ \mathcal{B}^{+T} & \mathcal{C} & -\mathcal{B}^{T} \\ \mathcal{A} & -\mathcal{B} & \mathbf{O}\end{array}\right], \quad \mathcal{M}\left[\begin{array}{l}h \\ w \\ \lambda\end{array}\right]=\left[\begin{array}{l}0 \\ 0 \\ b\end{array}\right]$

with $b^{T}=\left[b_{\mathcal{D}}^{T} \mathbf{O}^{T}\right]$. Well posedness of problem Eq. 22Eq. 24 derives from non singularity of the saddle point matrix $\mathcal{M}$.

Lemma 1 Let matrices $\mathcal{A}, \mathcal{B}$ be defined as in Eq. 25. Let $\mathcal{A}$ be full rank, let $\boldsymbol{L}=[\mathcal{A}-\mathcal{B}]$, and let $\boldsymbol{Z}$ be a matrix obtained collecting row-wise column vectors $z_{k}, k=1, \ldots, \mathcal{N}_{w}$, $\mathcal{N}_{w}=\mathcal{N}_{u}+\mathcal{N}_{q}$ forming a basis of $\operatorname{ker}(\boldsymbol{L})$, then matrix $\boldsymbol{Z}^{T} \boldsymbol{G Z}$ is positive definite.

Proof We start observing that matrix $\mathcal{A}$ is full rank as both matrices $\mathbf{A}_{\mathcal{D}}$ and $\mathbf{A}_{\mathcal{F}}$ are full rank under the assumption that $\alpha, \beta>0$. Then $\operatorname{dim}(\operatorname{ker}(\mathcal{L}))=\mathcal{N}_{w}$. To construct a basis of $\operatorname{ker}(\mathcal{L})$, let us take $e_{k}$, the $k$-th vector of the canonical basis of $\mathbb{R}^{\mathcal{N}_{w}}$, and let us set $z_{k}=\left(\mathcal{A}^{-1} \mathcal{B} e_{k}, e_{k}\right)$. According to the index $k, e_{k}$ might correspond to a non-null function $q_{i}$ for some $i=1, \ldots, N_{F}$ or a non-null function $u_{i}^{m}$ for some $i=1, \ldots, N_{F}, m \in \mathcal{S}_{i}$. In both cases will show that $z_{k}^{T} \mathbf{G} z_{k}>0$.

\section{Non-null function $q_{i}$}

Let us start considering the first case and in particular let us assume that $q_{i}=\varphi_{i, j}$ for a certain index $j=1, \ldots, \mathcal{N}_{q_{i}}$. 
Let us consider two different scenarios: the case $N_{F}=1$, i.e. a porous medium with a single fracture and the more general case $N_{F}>1$.

- $N_{F}=1$

As $q_{1} \neq 0$, for the non singularity of $\mathbf{A}_{\mathcal{D}}$, it is $h_{\mathcal{D}} \neq 0$, and in particular it can be either $h_{\mathcal{D}}=$ const or $h_{\mathcal{D}} \neq$ const.

- $h_{\mathcal{D}}=$ const $\neq 0$

As we assumed homogeneous Dirichlet conditions, $h_{\mathcal{D}}=$ const $\neq 0$ is possible only if $\Gamma_{D}=\emptyset$. In virtue of definition Eq. 11, for the consistency and conformity of the method, we have $\beta h_{\mathcal{D} \mid F_{1}}=q_{1}$, which is possible only if $q_{1}$ is constant on $F_{1}$. By Eq. 13, being $\left|\gamma_{1 D}\right|>0$, it is:

$$
\begin{aligned}
a_{F_{1}}\left(h_{1}, \psi_{1, \ell}\right) & =-b_{1}\left(q_{1}-\beta h_{\mathcal{D} \mid F_{1}}, \psi_{1, \ell}\right)=0, \\
\forall \ell & =1, \ldots, \mathcal{N}_{h_{1}}
\end{aligned}
$$

and thus $h_{1}=0$, and in particular $\| h_{\mathcal{D} \mid F_{1}}-$ $h_{1} \|_{\mathrm{L}^{2}\left(F_{1}\right)}>0$.

- $h_{\mathcal{D}} \neq$ const

Let us set $s_{1}:=q_{1}-\beta h_{\mathcal{D} \mid F_{1}}$, and let us consider equations 12 and 13 , that become:

$\left(\mathbf{K}_{\mathcal{D}} \nabla h_{\mathcal{D}}, \nabla \phi_{\ell}\right)_{\mathcal{D}}=b_{1}\left(s_{1}, \phi_{\ell \mid F_{1}}\right), \quad \forall \ell=1, \ldots, \mathcal{N}_{h_{\mathcal{D}}}$

$\left(\mathbf{K}_{i} \nabla_{i} h_{i}, \nabla_{i} \psi_{1, \ell}\right)_{F_{1}}=-b_{1}\left(s_{1}, \psi_{1, \ell}\right), \quad \forall \ell=1, \ldots, \mathcal{N}_{h_{1}}$ where the source term $s_{1}$ appears with opposite sign. We thus have $\left\|h_{\mathcal{D} \mid F_{1}}-h_{1}\right\|_{\mathrm{L}^{2}\left(F_{1}\right)}>0$.

- $\quad N_{F}>1$

If $h_{\mathcal{D}}=$ const $\neq 0$, proceeding similarly to the case $N_{F}=1$, we have $\beta h_{\mathcal{D} \mid F_{i}}=q_{i} \neq 0$ and $\| h_{\mathcal{D} \mid F_{i}}-$ $h_{i} \|_{\mathrm{L}^{2}\left(F_{i}\right)}>0$.

If instead $h_{\mathcal{D}} \neq$ const, we proceed in the following way: since $q_{i} \neq 0$ we have $h_{i} \neq 0$, whereas it is $h_{j}=0$, for all $j=1, \ldots, N_{F}, j \neq i$. Choosing, in particular, one index $j^{\star}$ such that fracture $F_{i}$ and $F_{j^{\star}}$ intersect in a trace $S_{m}$, we have $\left\|h_{i}-h_{j^{\star}}\right\|_{\mathrm{L}^{2}\left(S_{m}\right)}>0$.

Non-null function $u_{i}^{m}$

Let us now consider $u_{i}^{m}=\varrho_{i, j}^{m}$ for some $i=1, \ldots, N_{F}$, $m \in \mathcal{S}_{i}$, and for an index $j=1, \ldots, \mathcal{N}_{u_{i}}^{m}$, depending on the value of $k$. Also in this case it can be easily shown that we have $h_{i} \neq 0$, whereas we have $h_{\mathcal{D}}=0$ and $h_{p}=0$ for all $p=1, \ldots, N_{F}, p \neq i$, thus having again a non null functional value and thus $z_{k}^{T} \mathbf{G} z_{k}>0$.

Being $\mathbf{G}$ positive semi-definite by definition, it is $x^{T} \mathbf{G} x \geq 0$ and $x^{T} \mathbf{G} x=0$ if and only if $x \in \operatorname{ker}(\mathbf{G})$, [33] and being $z_{k}^{T} \mathbf{G} z_{k}>0, z_{k} \notin \operatorname{ker}(\mathbf{G})$ for $z=1, \ldots, \mathcal{N}_{w}$. The space $\mathcal{Z}=\operatorname{span}\left\{z_{1}, \ldots, z_{N_{w}}\right\}$ is thus a subspace of $\operatorname{Im}(\mathbf{G})$, and each vector $y \in \mathcal{Z}$ can be written as $y=\mathbf{Z} v$, for a vector $v \in \mathbb{R}^{\mathcal{N}_{w}}, v \neq 0$. Then $v^{T} \mathbf{Z}^{T} \mathbf{G Z} v>0$.
Theorem 1 Problem Eq. 26 has a unique solution $h^{\star}=$ $\left[\left(h_{\mathcal{D}}^{\star}\right)^{T},\left(h_{\mathcal{F}}^{\star}\right)^{T}\right]^{T}, w^{\star}=\left[\left(q^{\star}\right)^{T},\left(u^{\star}\right)^{T}\right]^{T}, \lambda^{\star}$, such that $h_{\mathcal{D}}^{\star}, h_{\mathcal{F}}^{\star}, q^{\star}, u^{\star}$ correspond to the constrained minimum of problem Eqs. 22-24.

The proof follows from Lemma 1 applying a classical argument of quadratic programming (see Theorem 16.2 in [34]).

\section{Unconstrained optimization problem}

We can proceed formally, replacing the constraint equations into the functional, to obtain an unconstrained minimization problem. We have $h=\mathcal{A}^{-1}(\mathcal{B} w+b)$, from which we obtain:

$$
\begin{aligned}
\mathcal{J}^{\star}(w)= & w^{T}\left(\mathcal{B}^{T} \mathcal{A}^{-T} \mathbf{G} \mathcal{A}^{-1} \mathcal{B}+\mathcal{C} y-\mathcal{B}^{T} \mathcal{A}^{-T} \mathcal{B}^{+}\right. \\
& \left.-\alpha \mathcal{B}^{+T} \mathcal{A}^{-1} \mathcal{B}\right) w \\
& +2\left(b^{T} \mathcal{A}^{-T} \mathbf{G} \mathcal{A}^{-1} \mathcal{B}-b^{T} \mathcal{A}^{-T} \mathcal{B}\right) w \\
& +b^{T} \mathcal{A}^{-T} \mathbf{G} \mathcal{A}^{-1} b \\
:= & w^{T} \mathcal{G} w+2 g w+\text { const }
\end{aligned}
$$

The unconstrained minimization problem then reads

$\min _{w} w^{T} \mathcal{G} w+2 g^{T} w$

or equivalently $\mathcal{G} w+g=0$. Matrix $\mathcal{G}$ is symmetric positive definite, given the equivalence of Eq. 27 with Eq. 22-Eq. 24. The unconstrained minimization problem can thus be solved with a gradient based iterative method, such as the conjugate gradient method. The steps of the method are as follows:

$$
\begin{aligned}
& \text { guess } w_{0} \\
& \text { compute } \gamma_{0}=\left(\mathcal{G} w_{0}+g\right) \text { and set } d_{0}=-\gamma_{0} \\
& \text { set } k=0 \\
& \text { while } \gamma_{k} \neq 0 \\
& \qquad \begin{array}{l}
\text { compute step size } \zeta_{k}=\frac{\gamma_{k}^{T} \gamma_{k}}{d_{k}^{T} \mathcal{G} d_{k}} \\
\text { set } w_{k+1}=w_{k}+\zeta_{k} d_{k} \\
\text { set } \gamma_{k+1}=\gamma_{k}+\zeta_{k} \mathcal{G} d_{k} \\
\text { compute } \theta_{k+1}=\frac{\gamma_{k+1}^{T} \gamma_{k+1}}{\gamma_{k}^{T} \gamma_{k}} \\
\text { set } d_{k+1}=-\gamma_{k+1}+\theta_{k+1} d_{k} \\
\text { set } k=k+1
\end{array}
\end{aligned}
$$

end

The computation of quantity $y_{k}=\mathcal{G} d_{k}$, at each step $k$ can be performed as follows: setting $\bar{h}_{k}=\mathcal{A}^{-1}\left(\mathcal{B} d_{k}\right)$ and $\lambda_{k}=\mathcal{A}^{-T}\left(\mathbf{G} \bar{h}_{k}-\mathcal{B}^{+} d_{k}\right)$, it is $y_{k}=\mathcal{B}^{T} \lambda_{k}+\mathcal{C} y d_{k}-\mathcal{B}^{+T} \bar{h}_{k}$. If $\beta=0$, which is possible as long as there is a non empty portion of the Dirichlet boundary for the three dimensional domain, i.e. $\left|\Gamma_{D}\right|>0$, the computation of $\bar{h}_{k}, \lambda_{k}$ at each step can be performed independently and in parallel on each 
fracture and on the three dimensional domain, thus easily allowing to use parallel computing resources for efficient resolution of the scheme, thanks to the block diagonal structure of $\mathcal{A}$ and $\mathbf{A}_{\mathcal{F}}$. If $\beta>0$ then problems on the fractures can be decoupled from the problem in the bulk domain as follows: at step $k>0$, being $\bar{h}_{k}=\left[\begin{array}{ll}\bar{h}_{\mathcal{D}, k}^{T} & \bar{h}_{\mathcal{F}, k}^{T}\end{array}\right]^{T}$ and splitting $d_{k}=\left[\begin{array}{ll}d_{q, k}^{T} & d_{u, k}^{T}\end{array}\right]^{T}$, we compute

$\bar{h}_{\mathcal{D}, k}=\mathbf{A}_{\mathcal{D}}^{-1}\left(\mathbf{E} d_{q, k}\right)$

$\bar{h}_{\mathcal{F}, k}=\mathbf{A}_{\mathcal{F}}^{-1}\left(\mathbf{B} d_{u, k}-\beta\left(\mathbf{G}^{\mathcal{D} F}\right)^{T} \bar{h}_{\mathcal{D}, k-1}-\mathbf{D} d_{q, k}\right)$,

and similarly for $\lambda_{k}$.

\section{Numerical results}

In this section we provide some numerical results in order to show the applicability of the present approach to flow simulations in porous media crossed by arbitrarily complex networks of fractures. All the simulations are performed using linear Lagrangian finite elements on $\mathcal{T}_{\delta_{\mathcal{D}}}^{\mathcal{D}}$ for $h_{\mathcal{D}}$, linear Lagrangian finite elements on $\mathcal{T}_{\delta_{F_{i}}}^{i}$ for $h_{i}$, piecewise constant basis functions on $\mathcal{T}_{\delta_{\Gamma_{i}}}^{i}$ for $q_{i}$ and piece-wise constant basis functions on $\mathcal{T}_{\delta_{S_{m}, i}^{i}}$ for $u_{i}^{m}$ on each trace $S_{m}$, on each fracture $F_{i}, i=1, \ldots, N_{F}, m \in \mathcal{S}_{i}$. In all cases we set $\beta=1$ and, if $\mathcal{S} \neq \emptyset, \alpha=1$, even if other choices of $\alpha, \beta>0$ could be equivalently used. Indeed we remark that the value of such parameters does not play any relevant role on the quality of the computed solution and values of $\alpha, \beta>0$ are only needed for well posedness of local problems.

\subsection{Problems with known solution}

We first propose two simple problems with known analytical solutions, labelled Problem 1 and Problem 2, having the same domain and type of boundary conditions. A cubic domain with unitary edge length is considered; the bottom face is on the plane $z=-\frac{1}{2}$ with respect to a reference system $\mathcal{O} x y z$, and the cube is crossed by a single fracture $F_{1}$ placed on the plane $z=0$, see Figure 3, left. The problems are set as follows:

$$
\begin{aligned}
& a_{\mathcal{D}}\left(H_{\mathcal{D}}, v\right)-b_{1}\left(Q_{1}, v_{\mid \Gamma_{i}}\right)=(f, v) \\
& a_{1}\left(H_{1}, w_{1}\right)+\left(H_{\mathcal{D} \mid F_{1}}, w_{1}\right)_{F_{1}}=-b_{1}\left(Q_{1}, w_{1}\right) \\
& b_{1}\left(H_{\mathcal{D} \mid F_{1}}-H_{1}, \mu_{1}\right)=0, \quad \forall \mu_{1} \in \mathrm{V}_{1}^{\prime}
\end{aligned}
$$

with $f=-1$ for Problem 1 and $f=0$ for Problem 2, $\mathbf{K}_{\mathcal{D}}=\mathbf{K}_{1}=1$ for both problems. Dirichlet boundary conditions are set on cube faces on planes $z=-\frac{1}{2}, z=\frac{1}{2}$, $x=0, x=1$, Neumann boundary conditions on cube faces on planes $y=0$ and $y=1$. Boundary conditions on fracture edges are prescribed accordingly to the boundary conditions on cube edges. Dirichlet and Neumann boundary conditions are derived from the analytical solution, which is $h=\frac{1}{4}\left(x^{2}+y^{2}\right)-\frac{1}{2}|z|$ for Problem 1 and $h=\frac{1}{2}\left(x^{2}-\right.$ $\left.y^{2}\right)+z$ for Problem 2. The two problems here considered also share the same meshes. In Figures 3, right and 4, we display the colormap of the solution of Problem 1. The mesh for the three dimensional domain is non conforming with the fracture plane and independent from the mesh on the fracture, as shown in Figure 3, right. In Figure 5 we report the behaviour of the error with respect to the mesh size both in $L^{2}$ and in $H^{1}$ norm for Problem 1. The three dimensional mesh parameter ranges between 0.02 and $4 \times 10^{-5}$, the mesh on the fracture between 0.3 and $5 \times 10^{-3}$. Due to the non conformity of the mesh and to the irregular behaviour of the solution across the interface, sub-optimal convergence trends are obtained. The obtained slopes for the error are compatible with the bounded regularity of the solution $h \notin H^{2}(\mathcal{D})$. The absolute value of the relative error for Problem 1 on the finest mesh is reported in Figure 6, showing that the error in the $3 \mathrm{D}$ solution is mainly located near the interface, where the solution is irregular and the mesh is not conforming with the irregularity interface. The error for the 2D solution appears, instead, almost equally distributed in the domain, going to zero towards the edges with Dirichlet boundary conditions. Optimal convergence curves are however recovered if the solution across the interface is smooth. In fact, if we consider Problem 2, having a smooth solution, optimal convergence trends are recovered, as reported in Figure 7. In this case, the error is smoothly distributed in both the 3D and 2D domains, as shown in Figure 8 , where the absolute value of the relative residual is reported for the 3D and 2D solutions of Problem 2.

\subsection{Simple DFN problem}

The second example proposes a validation of the method on a more general DFN configuration through a qualitative comparison of the obtained solution with the solution given by a method based on conforming meshes.

Let us consider a unitary edge cubic block of porous material, crossed by a network of 10 fractures forming 14 traces, as shown in Figure 9. A Dirichlet boundary condition equal to 1 is imposed on the bottom face of the cubic domain, whereas homogeneous Dirichlet boundary conditions are imposed on the edges of the fractures lying on the top face of the domain, being instead the top face and all other block faces and fracture edges insulated. The hydraulic trasmissivity is set to one for matrix and fractures. The same problem is solved with a VEM based approach on a polygonal/polyhedral conforming mesh, following the approach described in [23], and with the proposed FEM based optimization method. A fine mesh is used for the 
Fig. 3 Problem 1: domain description (left) and detail of the non conforming mesh at the interface (right)
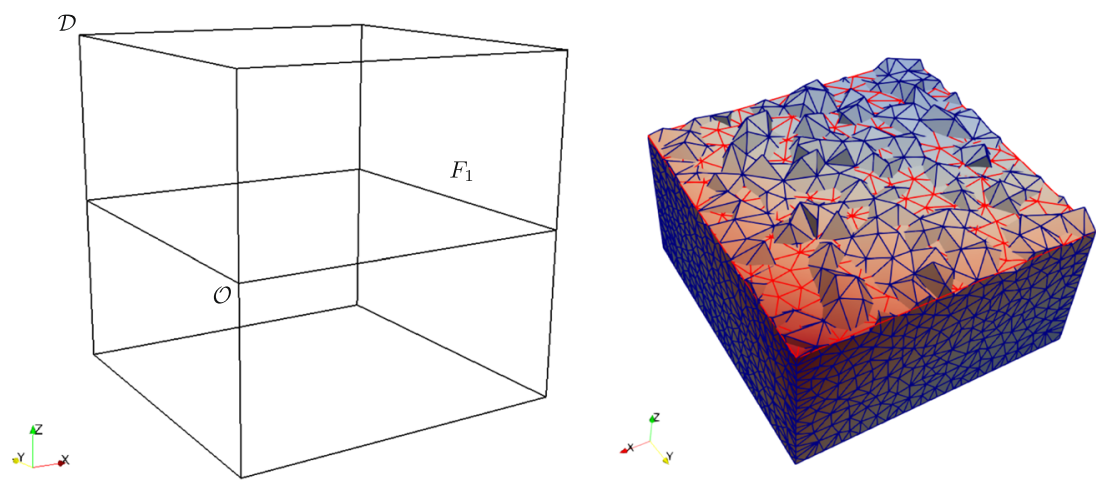

Fig. 4 Problem 1: solution on the three dimensional domain (left) and solution on the fracture (right)
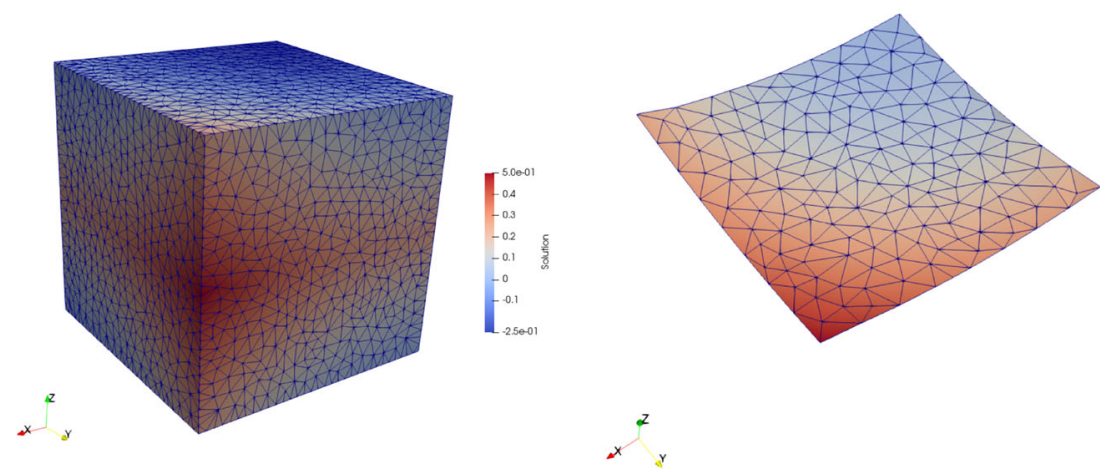
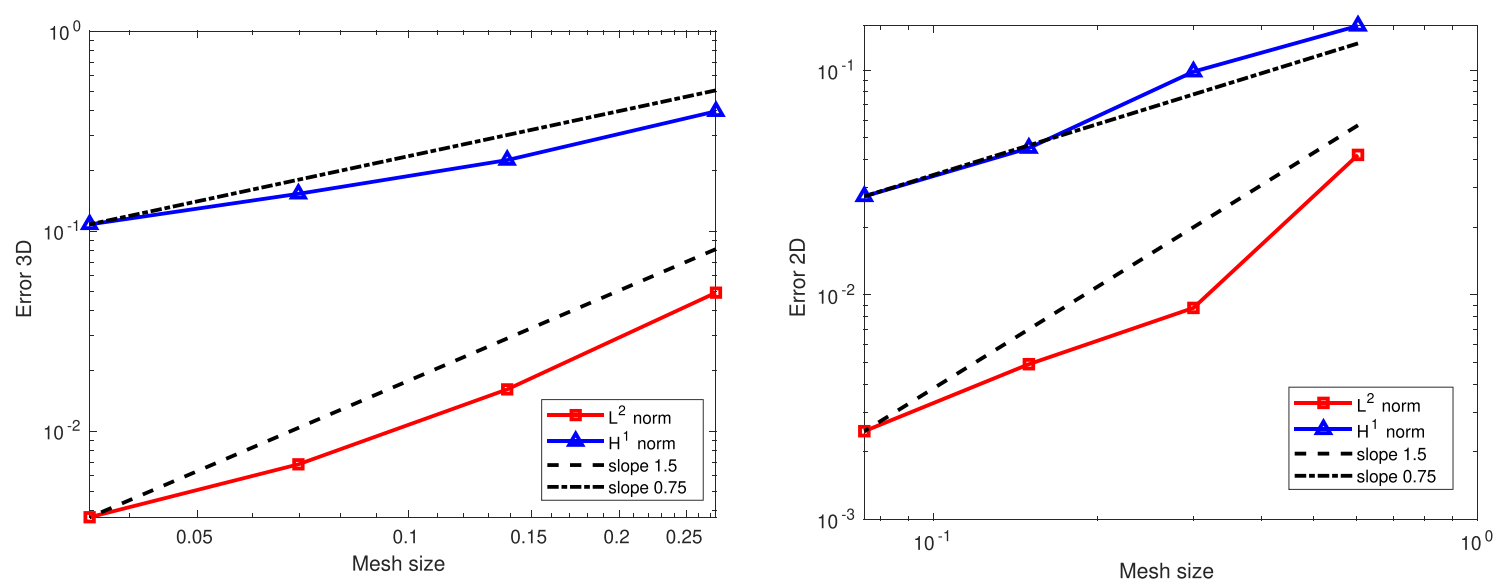

Fig. 5 Problem 1: convergence curves against mesh refinement

Fig. 6 Problem 1: Distribution of the absolute value of the relative error for the $3 \mathrm{D}$ and $2 \mathrm{D}$ solution on the finest mesh
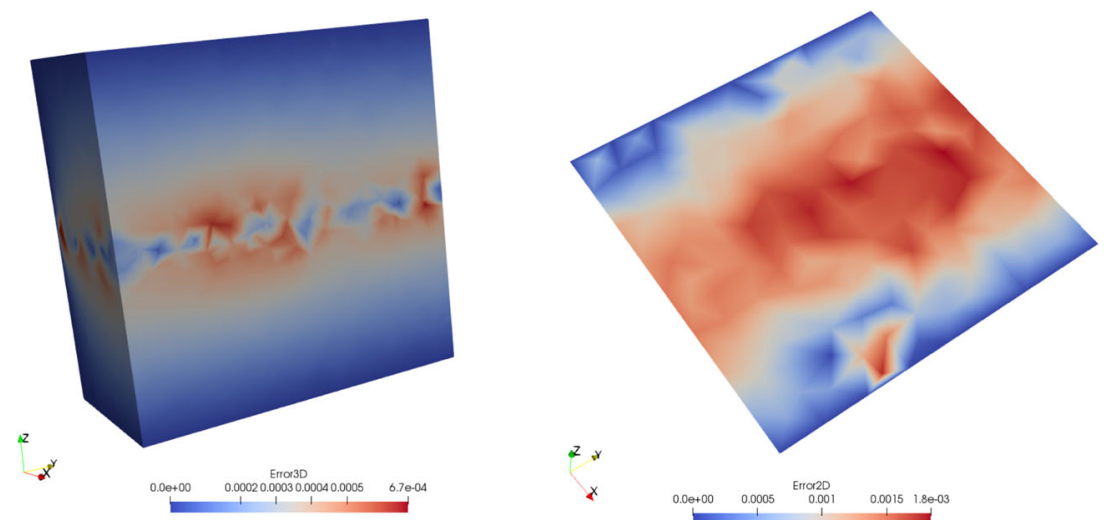

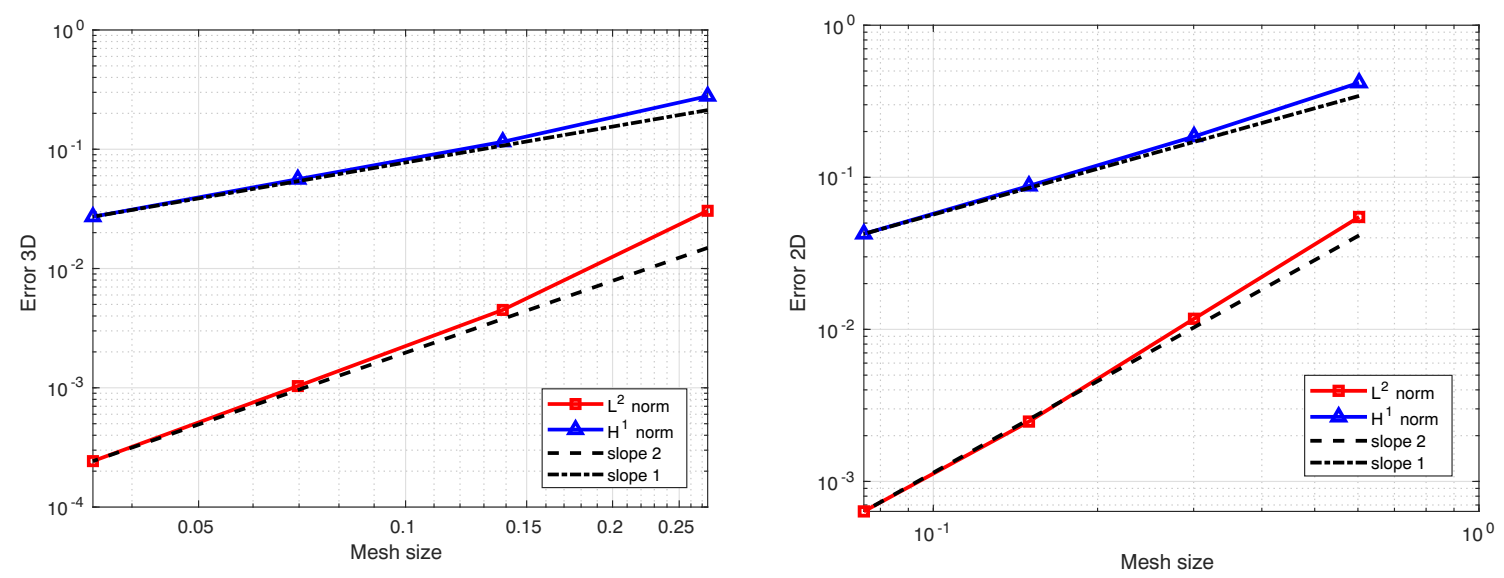

Fig. 7 Problem 2: convergence curves against mesh refinement for a problem with smooth solution across interface

Fig. 8 Problem 2: Distribution of the absolute value of the relative error for the $3 \mathrm{D}$ and $2 \mathrm{D}$ solution on the finest mesh
Fig. 9 Problem 3:

Computational domain, mesh and solution with the VEM on conforming meshes (left) and with the FEM based optimization method on non conforming meshes (right)
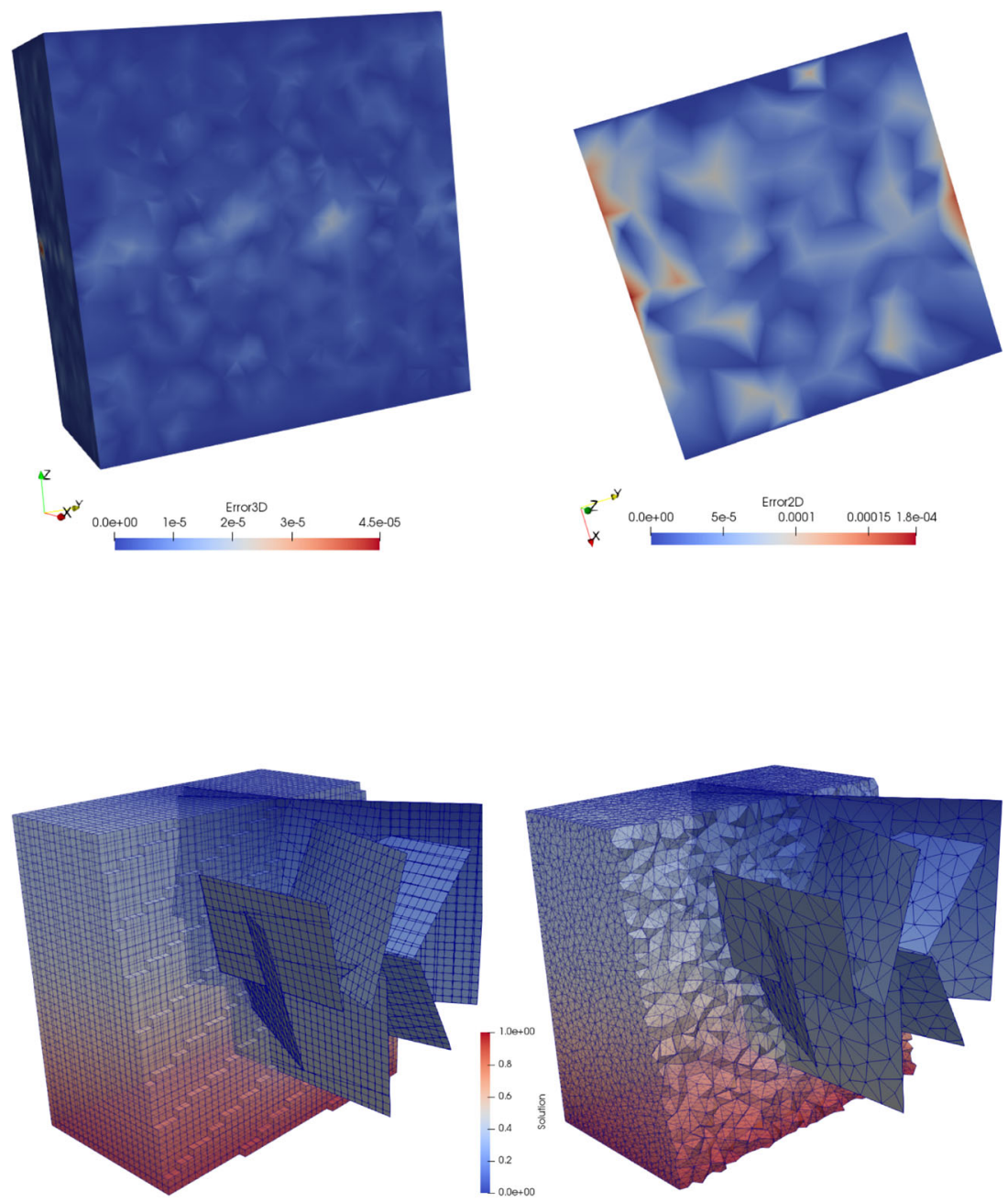


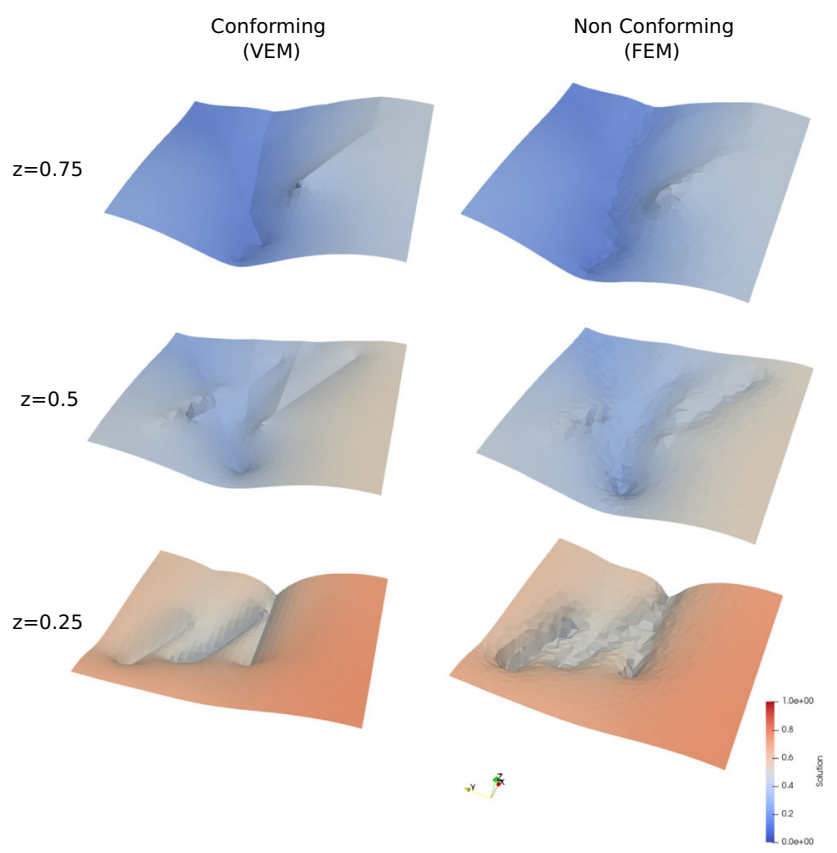

Fig. 10 Problem 3: Qualitative comparison of the solution obtained with the VEM on conforming meshes (left) and with the FEM based optimization method on non conforming meshes (right)

VEM based reference solution and for the optimization based solution, with elements of maximum size of about $1.5 \times 10^{-5}$, shown again in Figure 9. The solution obtained with the two approaches is reported in Figure 10 on three different planes, placed at $z=0.25, z=0.5$, and $z=0.75$, on the left for the VEM based approach on the conforming mesh and on the right for the FEM based optimization approach on non conforming meshes. We can see that the two solutions are in very good agreement, despite the irregularities of the solution are not reproduced on the non conforming mesh as sharply as it is done on the conforming mesh. On the other hand, the optimization approach allows an easy and robust meshing process, that ensures the generation of good shaped elements independently of the complexity of the geometry.

\subsection{DFN problem}

In the last example, a more complex and realistic DFN is considered, embedded in a cubic domain, with barycentre in the origin of a reference system $\mathcal{O} x y z$ and edge length equal to two, as shown in Figure 11, on the left. The embedded DFN consists of 20 randomly placed fractures, forming 62 traces, with a number of traces per fracture ranging between 4 and 10. Traces intersect, forming angles as narrow as 11.5 degrees, whereas the minimum angle between the normals of couples of intersecting fractures is 17.3 degrees. A unitary pressure Dirichlet boundary condition is imposed on fracture edges lying on the planes $z=1$, and a zero pressure Dirichlet boundary condition is imposed on the cube face on the plane $z=0$, all other fracture edges and cube faces being insulated. An inflow is thus obtained through some fracture edges, and outflow occurs through the bottom face of the cube. Figure 11, on the right, shows the computed solution on the 3D domain and on the fractures, through a section of the three dimensional domain, along with the used mesh, characterized by a mesh parameter equal to $\delta_{\mathcal{D}}=0.001$ for the tetrahedral mesh and to $\delta_{F_{i}}=0.05$, $i=1, \ldots, 20$ for the triangular mesh on all the fractures. On this mesh, the total number of unknowns, $\mathcal{N}_{h}+\mathcal{N}_{q}+\mathcal{N}_{u}$ is 5541, and the minimization problem is solved using 90 iterations to reach a relative residual of $10^{-8}$, resulting in a functional value of 0.069 . Considering a refined mesh, with mesh parameter $1.25 \times 10^{-4}$ for the tetrahedral mesh and $1.25 \times 10^{-2}$ for the triangular mesh, the total number of unknowns rises to 28686 and the number of iterations to reach the same relative residual is 125 and the functional value is reduced to 0.057 . We remark that the minimum of the discrete functional is greater than zero, as a consequence of the non conformity of the mesh. These results show the viability of the proposed approach in dealing with complex domains.

\section{Conclusions}

A new discretization strategy for the simulation of the flow in arbitrarily complex DFM geometries has been presented
Fig. 11 DFN problem: domain (left) and a section of the computed solution (right)
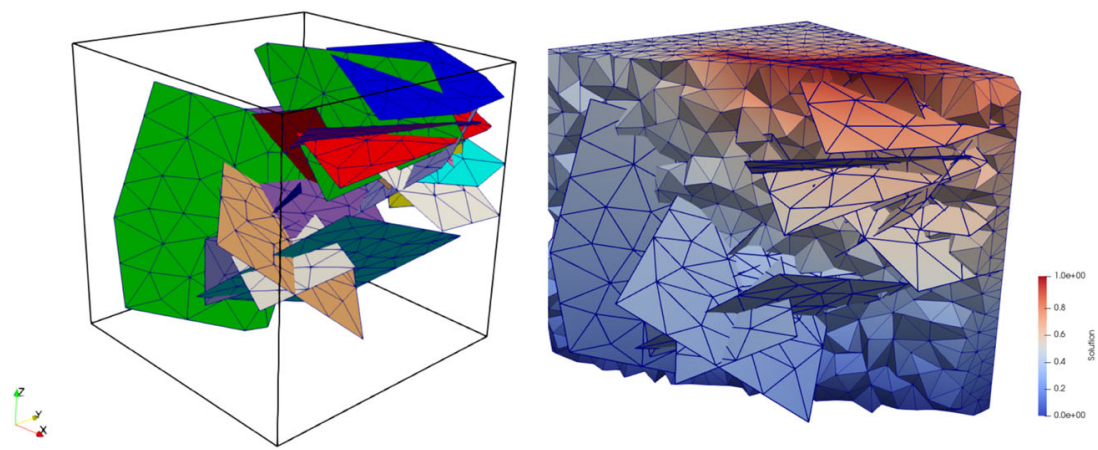
and validated. The method is based on standard finite element discretizations for both the three dimensional domain and the fractures, and the meshing can be performed independently on each geometrical entity, thus actually overcoming any mesh related issue for DFM simulations. The resulting discrete problem is well posed and can be efficiently solved via a gradient scheme. The proposed numerical tests validate the method and show its applicability to realistic DFM configurations. Although the proposed method can be easily implemented for parallel solution, optimal parallel solver and suitable well balanced partitioning strategies, yielding to efficient parallel solvers, should be investigated but are out of the scope of the present work.

Funding Open Access funding provided by Politecnico di Torino.

Acknowledgements This work was supported by the MIUR project "Dipartimenti di Eccellenza 2018-2022" (CUP E11G18000350001), PRIN project "Virtual Element Methods: Analysis and Applications" (201744KLJL_004), by INdAM-GNCS and by SmartData@polito.

Open Access This article is licensed under a Creative Commons Attribution 4.0 International License, which permits use, sharing, adaptation, distribution and reproduction in any medium or format, as long as you give appropriate credit to the original author(s) and the source, provide a link to the Creative Commons licence, and indicate if changes were made. The images or other third party material in this article are included in the article's Creative Commons licence, unless indicated otherwise in a credit line to the material. If material is not included in the article's Creative Commons licence and your intended use is not permitted by statutory regulation or exceeds the permitted use, you will need to obtain permission directly from the copyright holder. To view a copy of this licence, visit http:// creativecommonshorg/licenses/by/4.0/.

\section{References}

1. Martin, V., Jaffré, J., Roberts, J.E.: Modeling fractures and barriers as interfaces for flow in porous media. SIAM J. Sci. Comput. 26(5), 1667-1691 (2005). https://doi.org/10.1137/ S1064827503429363

2. Boon, W., Nordbotten, J., Yotov, I.: Robust discretization of flow in fractured porous media. SIAM J. Numer. Anal. 56(4), 22032233 (2018). https://doi.org/10.1137/17M1139102

3. Qi, D., Hesketh, T.: An analysis of upscaling techniques for reservoir simulation. Pet. Sci. Technol. 23(7-8), 827-842 (2005). https://doi.org/10.1081/LFT-200033132

4. Chen, Z., Huan, G., Ma, Y.: Computational methods for multiphase flows in porous media. SIAM, Philadelphia, PA, USA (2006)

5. Li, L., Lee, S.H.: Efficient field-scale simulation of black oil in a naturally fractured reservoir through discrete fracture networks and homogenized media. SPE Reservoir Evaluation \& Engineering 11(4), 750-758 (2008). https://doi.org/10.2118/103901-PA

6. Moinfar, A., Varavei, A., Sepehrnoori, K., Johns, R.: Development of an efficient embedded discrete fracture model for $3 \mathrm{~d}$ compositional reservoir simulation in fractured reservoirs. SPE J., 19(2) (2014)

7. Fumagalli, A., Pasquale, L., Zonca, S., Micheletti, S.: An upscaling procedure for fractured reservoirs with embedded grids. Water Resour. Res. 52(8), 6506-6525 (2015). https://doi.org/10.1002/ 2015WR017729
8. Angot, P., Boyer, F., Hubert, F.: Asymptotic and numerical modelling of flows in fractured porous media. ESAIM: M2AN 43(2), 239-275 (2009)

9. Ahmed, R., Edwards, M.G., Lamine, S., Huisman, B.A.H., Pal, M.: Control-volume distributed multi-point flux approximation coupled with a lower-dimensional fracture model. J. Comput. Phys. 284, 462-489 (2015). https://doi.org/10.1016/j.jcp.2014.12. 047

10. Brenner, K., Groza, M., Guichard, C., Lebeau, G., Masson, R.: Gradient discretization of hybrid dimensional darcy flows in fractured porous media. Numer. Math. 134(3), 569-609 (2016). https://doi.org/10.1007/s00211-015-0782-x

11. Antonietti, P.F., Formaggia, L., Scotti, A., Verani, M., Verzott, N.: Mimetic finite difference approximation of flows in fractured porous media. ESAIM: M2AN 50(3), 809-832 (2016)

12. Berrone, S., Pieraccini, S., Scialò, S.: Flow simulations in porous media with immersed intersecting fractures. J. Comput. Phys. 345, 768-791 (2017). https://doi.org/10.1016/j.jcp.2017.05.049

13. Odsæter, L.H., Kvamsdal, T., Larson, M.G.: A simple embedded discrete fracture-matrix model for a coupled flow and transport problem in porous media. Comput. Methods Appl. Mech. Eng. 343, 572-601 (2019). https://doi.org/10.1016/j.cma.2018.09.003

14. Antonietti, P.F., Facciolà, C., Russo, A., Verani, M.: Discontinuous Galerkin approximation of flows in fractured porous media on polytopic grids. SIAM J. Sci. Comput. 41(1), A109-A138 (2019). https://doi.org/10.1137/17M1138194

15. Chernyshenko, A.Y., Olshanskii, M.A.: An unfitted finite element method for the darcy problem in a fracture network. J. Comput. Appl. Math. 366, 112424 (2020). https://doi.org/https://doi.org/10. 1016/j.cam.2019.112424

16. Fumagalli, A., Scotti, A.: A numerical method for two-phase flow in fractured porous media with non-matching grids. Adv. Water Resour. 62, 454-464 (2013). https://doi.org/10.1016/j.advwatres. 2013.04.001

17. Formaggia, L., Fumagalli, A., Scotti, A., Ruffo, P.: A reduced model for Darcy's problem in networks of fractures. ESAIM: Mathematical Modelling and Numerical Analysis 48(4), 10891116 (2014007). https://doi.org/10.1051/m2an/2013132

18. Lipnikov, K., Manzini, G., Shashkov, M.: Mimetic finite difference method. J. Comput. Phys. 257, 1163-1227 (2014)

19. Al-Hinai, O., Srinivasan, S., Wheeler, M.F.: Mimetic finite differences for flow in fractures from microseismic data. In: SPE reservoir simulation symposium. Society of Petroleum Engineers (2015)

20. Chave, F.A., Di Pietro, D.A., Formaggia, L.: A Hybrid HighOrder method for Darcy flows in fractured porous media. SIAM Journal on Scientific Computing 40(2), A1063-A1094 (2018). https://doi.org/10.1137/17M1119500

21. Sandve, T.H., Berre, I., Nordbotten, J.M.: An efficient multipoint flux approximation method for discrete fracture-matrix simulations. J. Comput. Phys. 231(9), 3784-3800 (2012). https://doi.org/10.1016/j.jcp.2012.01.023

22. Faille, I., Fumagalli, A., Jaffré, J., Roberts, J.E.: Model reduction and discretization using hybrid finite volumes for flow in porous media containing faults. Comput. Geosci. 20(2), 317-339 (2016). https://doi.org/10.1007/s10596-016-9558-3

23. Berrone, S., Borio, A., Fidelibus, C., Pieraccini, S., Scialò, S., Vicini, F.: Advanced computation of steady-state fluid flow in discrete fracture-matrix models: FEM-BEM and VEMVEM fracture-block coupling. GEM - International Journal on Geomathematics 9(2), 377-399 (2018). https://doi.org/10.1007/ s13137-018-0105-3

24. Fumagalli, A., Keilegavlen, E.: Dual virtual element methods for discrete fracture matrix models. Oil Gas Sci. Technol. - Rev. IFP Energies nouvelles 74, 41 (2019) 
25. Coulet, J., Faille, I., Girault, V., Guy, N., Nataf, F.: A fully coupled scheme using virtual element method and finite volume for poroelasticity. Comput. Geosci. (2019)

26. Benedetto, M.F., Borio, A., Kyburg, F., Mollica, J., Scialò, S.: An arbitrary order Mixed Virtual Element formulation for coupled multi-dimensional flow problems. arXiv:2001.11309 (2020)

27. Berrone, S., Pieraccini, S., Scialò, S.: A PDE-constrained optimization formulation for discrete fracture network flows. SIAM J. Sci. Comput. 35(2), B487-B510 (2013). https://doi.org/10.1137/ 120865884

28. Berrone, S., Pieraccini, S., Scialò, S.: On simulations of discrete fracture network flows with an optimization-based extended finite element method. SIAM J. Sci. Comput. 35(2), A908-A935 (2013). https://doi.org/10.1137/120882883

29. Berrone, S., Borio, A., Scialò, S.: A posteriori error estimate for a PDE-constrained optimization formulation for the flow in DFNs. SIAM J. Numer. Anal. 54(1), 242-261 (2016). https://doi.org/10.1137/15M1014760
30. Berrone, S., Scialò, S., Vicini, F.: Parallel meshing, discretization and computation of flow in massive Discrete Fracture Networks. SIAM J. Sci. Comput. 41(4), C317-C338 (2019). https://doi.org/10.1137/18M1228736

31. Berrone, S., Borio, A., Vicini, F.: Reliable a posteriori mesh adaptivity in discrete fracture network flow simulations. Comput. Methods Appl. Mech. Eng. 354, 904-931 (2019). https://doi.org/10.1016/j.cma.2019.06.007

32. Berrone, S., D'Auria, A., Vicini, F.: Fast and robust flow simulations in discrete fracture networks with GPGPUs. GEM International Journal on Geomathematics 10(1), 8 (2019)

33. Horn, R.A., Johnson, C.R.: Matrix analysis. Cambridge University Press, Cambridge (1990)

34. Nocedal, J., Wright, S.J. Numerical optimization, Second. Springer, New York (2006)

Publisher's note Springer Nature remains neutral with regard to jurisdictional claims in published maps and institutional affiliations. 